Journal of

Applied

Crystallography

ISSN 0021-8898

Editor: Anke R. Pyzalla

\title{
Phase behaviour and thermoelastic properties of perdeuterated ammonia hydrate and ice polymorphs from 0 to $2 \mathrm{GPa}$
}

\author{
A. D. Fortes, I. G. Wood, L. Vočadlo, K. S. Knight, W. G. Marshall, M. G. \\ Tucker and F. Fernandez-Alonso
}

J. Appl. Cryst. (2009). 42, 846-866

Copyright (C) International Union of Crystallography

Author(s) of this paper may load this reprint on their own web site or institutional repository provided that this cover page is retained. Republication of this article or its storage in electronic databases other than as specified above is not permitted without prior permission in writing from the IUCr.

For further information see http://journals.iucr.org/services/authorrights.html

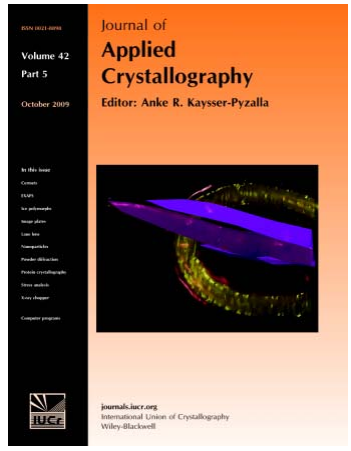

Many research topics in condensed matter research, materials science and the life sciences make use of crystallographic methods to study crystalline and non-crystalline matter with neutrons, X-rays and electrons. Articles published in the Journal of Applied Crystallography focus on these methods and their use in identifying structural and diffusioncontrolled phase transformations, structure-property relationships, structural changes of defects, interfaces and surfaces, etc. Developments of instrumentation and crystallographic apparatus, theory and interpretation, numerical analysis and other related subjects are also covered. The journal is the primary place where crystallographic computer program information is published.

Crystallography Journals Online is available from journals.iucr.org 
Journal of

Applied

Crystallography

ISSN 0021-8898

Received 16 April 2009

Accepted 15 July 2009

(C) 2009 International Union of Crystallography

Printed in Singapore - all rights reserved

\section{Phase behaviour and thermoelastic properties of perdeuterated ammonia hydrate and ice polymorphs from 0 to $2 \mathrm{GPa}$}

\author{
A. D. Fortes, ${ }^{a, b *}$ I. G. Wood, ${ }^{a, b}$ L. Vočadlo, ${ }^{a, b}$ K. S. Knight, ${ }^{c, d}$ W. G. Marshall, ${ }^{c}$ \\ M. G. Tucker ${ }^{c}$ and F. Fernandez-Alonso ${ }^{c}$ \\ ${ }^{a}$ Centre for Planetary Sciences at University College London/Birkbeck, Gower Street, London WC1E \\ 6BT, UK, ${ }^{\mathbf{b}}$ Department of Earth Sciences, University College London, Gower Street, London WC1E \\ 6BT, UK, 'ISIS Facility, STFC Rutherford Appleton Laboratory, Harwell Science and Innovation \\ Campus, Chilton, Didcot, Oxfordshire OX11 0QX, UK, and ${ }^{\mathbf{d}}$ The Natural History Museum, \\ Cromwell Road, London SW7 5BD, UK. Correspondence e-mail: andrew.fortes@ucl.ac.uk
}

\begin{abstract}
The results are described of a series of neutron powder diffraction experiments over the pressure and temperature ranges $0<P<2 \mathrm{GPa}, 150<T<240 \mathrm{~K}$, which were carried out with the objective of determining the phase behaviour and thermoelastic properties of perdeuterated ammonia dihydrate $\left(\mathrm{ND}_{3} \cdot 2 \mathrm{D}_{2} \mathrm{O}\right)$. In addition to the low-pressure cubic crystalline phase, ADH I, two closely related monoclinic polymorphs of ammonia dihydrate have been identified, which commonly occur as a composite in the range $450-550 \mathrm{MPa}$ at $175 \mathrm{~K}$; these are labelled $\mathrm{ADH}$ II $a$ and II $b$, and each has unit-cell volume $V \simeq 310 \AA^{3}$ and number of formula units per unit cell $Z=4$. It has been determined that this composite dissociates to a mixture of ammonia monohydrate $\left(\mathrm{ND}_{3} \cdot \mathrm{D}_{2} \mathrm{O}\right)$ phase II (AMH II) and ice II when warmed to $\sim 190 \mathrm{~K}$ at $550 \mathrm{MPa}$, which in turn partially melts to ice II + liquid at $T=196 \mathrm{~K}$; AMH II has a large orthorhombic unit cell ( $V \simeq 890 \AA^{3}, Z=16$ ). Above $600 \mathrm{MPa}$, an orthorhombic polymorph of ammonia dihydrate (with $V \simeq 530 \AA^{3}, Z=8$ ), which has been referred to previously as ADH IV, persists to pressures greater than $2 \mathrm{GPa}$ and appears to be the liquidus phase over this whole pressure range. This phase has been observed co-existing with ice II, ice VI and AMH II. The most plausible synthesis of the high-pressure phase behaviour is described here. This model explains the reported observations, and provides measurements of the densities, thermal expansion, bulk moduli and crystal growth kinetics of the high-pressure ammonia dihydrate, ammonia monohydrate and ice polymorphs.
\end{abstract}

\section{Introduction}

The water-ammonia system has long been of considerable interest to planetary scientists, since astronomical observations and cosmochemical models indicate that ammonia may be a significant component of the outer solar system volatile inventory (e.g. Lewis, 1971, 1972; Lewis \& Prinn, 1980; Prinn \& Fegley, 1981). Although there is some difference of opinion regarding the ammonia yield from various condensation models (Mousis, Gautier \& Bocklée-Morvan, 2002), and indeed the fate of that ammonia once accreted into large bodies such as Titan (Fortes, Grindrod et al., 2007; Grindrod et al., 2008), it remains amongst the most plausible planetary 'anti-freeze' agents, and its physical properties under the appropriate conditions (roughly 0-5 GPa, 100-300 K) must be known in order for it to be accommodated in planetary models. The pressure melting curve and the expected polymorphism of the stoichiometric ammonia hydrates have implications for the internal structure of large icy moons like
Titan, leading to phase layering and the possible persistence of deep subsurface oceans (Grasset \& Sotin, 1996a,b; Grasset et al., 2000; Sohl et al., 2003; Grasset \& Pargamin, 2005; Tobie et al., 2005; Lorenz et al., 2008), the latter being sites of high astrobiological potential (Fortes, 2000; Simakov, 2001; Raulin, 2008). Aqueous ammonia is also a candidate substance involved in cryomagmatism on Titan (Kargel, 1992; Lopes et al., 2007), and again the melting behaviour, and densities of liquids and solids, in the ammonia-water system must be known to model properly the partial melting and propagation of magma through a planetary crust. Lately, experimental studies of the water-ammonia-methane system have begun (Kurnosov et al., 2006; Choukroun et al., 2007), and proper interpretation of the ternary system must rely on an accurate description of the binary water-ammonia system at high pressures.

The water-ammonia system is also of interest to physical chemists for its mixture of homonuclear and heteronuclear hydrogen bonds. The end member phases and the stoichio- 
metric hydrates, ammonia dihydrate $\left(\mathrm{NH}_{3} \cdot 2 \mathrm{H}_{2} \mathrm{O}, \mathrm{ADH}\right)$, ammonia monohydrate $\left(\mathrm{NH}_{3} \cdot \mathrm{H}_{2} \mathrm{O}, \mathrm{AMH}\right)$ and ammonia hemihydrate $\left(\mathrm{NH}_{3} \cdot 0.5 \mathrm{H}_{2} \mathrm{O}, \mathrm{AHH}\right)$, are therefore model systems for understanding the behaviour of bonds that occur in far more complex molecules, such as DNA. Since the rich polymorphism and physical properties of water ices continue to shed new light on the behaviour of the homonuclear hydrogen bond between water molecules in the solid state (Zheligovskaya \& Malenkov, 2006), we can expect to learn more about mixed hydrogen bonds from studying the phase behaviour and physical properties of ammonia hydrates under high pressures.

The behaviour of ammonia dihydrate and ammonia monohydrate at high pressure has been investigated extensively over the past 25 years, for the most part by Raman and optical methods focused upon establishing the high-pressure melting curves (Johnson et al., 1984, 1985; Johnson \& Nicol, 1987; Croft et al., 1988; Cynn et al., 1989; Boone \& Nicol, 1991; Hogenboom \& Kargel, 1990; Hogenboom et al., 1989, 1994; Grasset \& Sotin, 1996b; Leliwa-Kopystiński et al., 2002; Mousis, Pargamin et al., 2002; Pargamin et al., 2002). Despite the observation that $\mathrm{ADH}$ crystals grown under high pressure do not exhibit the cubic symmetry of the low-pressure phase (Boone, 1989), the general conclusion of the early diamond anvil cell studies was that neither ADH nor AMH exhibited evidence of high-pressure polymorphism (Boone, 1989; Koumvakalis, 1988). However, dilatometric studies subsequently established the existence of a new high-pressure polymorph in each system, ADH II stable above $\sim 400 \mathrm{MPa}$ at $170 \mathrm{~K}$, and AMH II stable above $340 \mathrm{MPa}$ at $195 \mathrm{~K}$ (Kargel \& Hogenboom, 1995; Hogenboom et al., 1995, 1997). Shortly afterwards, neutron powder diffraction patterns of both phases were reported (Nelmes \& Loveday, 1998, 1999) from studies using a gas pressure cell on the POLARIS diffractometer at ISIS. AMH has since been shown to possess a number of high-pressure polymorphs (Loveday \& Nelmes, 1999, 2004).

We have carried out computational and experimental studies of ammonia hydrates, including a number of highpressure studies of ammonia dihydrate (Fortes, Wood, Brodholt \& Vočadlo, 2003; Fortes, Wood, Knight et al., 2003; Fortes, Wood et al., 2007; Fortes, 2004). Most recently, we summarized our high-pressure neutron diffraction studies of ADH (Fortes, Wood et al., 2007). In that paper, we stated that we had identified four high-pressure phases of $\mathrm{ADH}$; however, one of these phases (ADH III) does not in fact exist; it is actually a mixture of ammonia monohydrate II and ice II. Interestingly, this conclusion had been arrived at previously (Fortes, Wood, Knight et al., 2003; Fortes, 2004), but subsequent - flawed analysis led us to conclude later that this material was a single phase of ADH.

The first, often problematic, stage in the solution of unknown crystal structures from neutron powder diffraction data is one of indexing, the determination of the unit-cell dimensions. Powder indexing is a notoriously fickle enterprise with even the best data, particularly for large and/or lowsymmetry unit cells owing to the near equivalence of many reflections in one-dimensional powder patterns (see Bergmann et al., 2004) or owing to metric singularities (e.g. Mighell \& Santora, 1975). In our work we have met both large and low-symmetry unit cells, and have been faced with complex multiphase patterns (often containing three or four unidentified phases), frequently with many unwanted Bragg reflections due to the sample environment (cryostats and pressure cells). In some cases, specimens have disproportionated into two separate phases, and on one occasion glassy residue has crystallized, adding diffraction peaks to the pattern that were not previously present. The solution to this problem is to compare diffraction data collected across many experiments which we do here - observing closely the behaviour of all reflections through phase transitions and properly correcting for the sample environment. For example, we had argued (Fortes, Wood et al., 2007) that 'ADH III' was a single phase because it melted congruently, which is a facile argument since a mixture of phases will also melt simultaneously if the bulk composition corresponds to that of the eutectic. As shown below, proper subtraction of the sample environment contribution (which had not been done previously for the data recorded during this melting transition) reveals weak ice II peaks persisting to much higher temperatures, confirming that partial melting of a mixture had occurred. Through this more careful analysis we have now indexed (i.e. identified) all of the phases we have observed.

\section{Experimental method}

\subsection{Neutron powder diffractometers}

All of the neutron powder diffraction studies described in this paper were carried out at the ISIS neutron spallation source (Rutherford Appleton Laboratory, Chilton, UK) using the time-of-flight method. We have used three different instruments at ISIS - HRPD, OSIRIS and PEARL/HiPr each of which has different characteristics in terms of resolution and $d$-spacing range coverage. The highest-resolution diffractometer at ISIS, HRPD (Ibberson et al., 1992), has a $95 \mathrm{~m}$-long flight path viewing a $100 \mathrm{~K}$ liquid methane moderator. The bandwidth of the incident neutron pulse provides a typical time-of-flight (t-o-f) window of width $100 \mathrm{~ms}$. Note that the HRPD guide ${ }^{\mathbf{1}}$ is so long that the frameoverlap problem limits the instrument to using one pulse in five (i.e. $10 \mathrm{~Hz}$ incident beam). The other instruments used here - OSIRIS and PEARL/HiPr, with 35.0 and $12.6 \mathrm{~m}$ flight paths, respectively - utilize flight time windows 40 and $20 \mathrm{~ms}$ wide at 25 and $50 \mathrm{~Hz}$ pulse frequencies, respectively. The range of $d$ spacings measured in these windows depends on which range of flight times is chosen and the Bragg angle range of the detectors. On HRPD it is customary to select the 30 $130 \mathrm{~ms}$ t-o-f window, which allows measurement of $d$ spacings from 0.60 to $2.69 \AA$ in the backscattering detectors $(160<2 \theta<$ $176^{\circ}$, resolution $\left.\Delta d / d=4 \times 10^{-4}\right), 0.87-3.77 \AA$ in the $' 90^{\circ}$, detectors $\left(80<2 \theta<100^{\circ}, \Delta d / d=2 \times 10^{-3}\right)$ and $2.30-9.88 \AA$ in

\footnotetext{
${ }^{1}$ All of these measurements were made prior to the installation of HRPD's elliptical guide mirror in 2007.
} 
the low-angle detectors $\left(28<2 \theta<32^{\circ}, \Delta d / d=2 \times 10^{-2}\right)$. It is possible to select a single $200 \mathrm{~ms}$ t-o-f window to measure a very wide $d$-spacing histogram, at the expense of halving the count rate $(5 \mathrm{~Hz}$ pulse frequency); here we have counted in two separate $100 \mathrm{~ms}$ windows (30-130 and 100-200 ms) consecutively, at the instrument's normal $10 \mathrm{~Hz}$ frequency.

OSIRIS (Telling \& Andersen, 2005, 2008) views a $20 \mathrm{~K}$ liquid hydrogen moderator and thus receives a very high flux of colder (longer wavelength) neutrons, making it well suited to long $d$-spacing measurements. OSIRIS has a series of usercontrolled chopper phasings which select different flight time (or $d$-spacing) windows, each with the same neutron bandwidth but a different central wavelength. For example, $d$ range 1 covers the time window $11.7-51.7 \mathrm{~ms}$, yielding a histogram with $d$ spacings from 0.7 to $2.9 \AA$ in the backscattering detectors $\left(150<2 \theta<171^{\circ}\right.$, resolution $\left.\Delta d / d=6 \times 10^{-3}\right) ; d$ range 2 covers the time window 29.4-69.4 ms (1.8-4.0 $\AA$ ); $d$ range 3 covers $47.1-87.1 \mathrm{~ms}(2.9-4.9 \AA)$ and so on: in principle, one can examine $d$ spacings as large as $20 \AA$ on OSIRIS with the appropriate chopper phasing. Higher resolutions are possible by restricting the data to the fraction of detectors at highest $2 \theta$, and we have been able to achieve resolutions that compare very favourably with HRPD's $90^{\circ}$ banks $\left(\sim 2 \times 10^{-3}\right)$ using only the $1-5 \%$ of detectors at the highest scattering angles.

The PEARL beamline's HiPr diffractometer (see ISIS Annual Report, 1996) is equipped as a dedicated high-pressure facility, and its detector banks are arranged so as to take advantage of the restricted region of reciprocal space accessible inside the opposed anvil Paris-Edinburgh press. The transverse detectors $\left(83<2 \theta<97^{\circ}, \Delta d / d=8 \times 10^{-3}\right)$ typically used in 'through anvil' diffraction geometry allow measurement of $d$ spacings in the range $0.7-4.1 \AA$ with an effective useful time window extending from 3.3 to $19.3 \mathrm{~ms}$.

\subsection{Sample environment}

Bailey (2003) gives a general review of high-pressure sample environments in neutron scattering. High-pressure neutron diffraction investigations in the range $0.1-550 \mathrm{MPa}$ were carried out in aluminium 7075 alloy or $\mathrm{Ti}_{66} \mathrm{Zr}_{34}$ nullscattering alloy gas cells (sample volume $=1.539 \mathrm{~cm}^{3}$ ) using helium as the pressure-transmitting medium (see $\$ 5.1$ for discussion of the merits of using helium on ice-bearing specimens). Whilst considerably less absorbing than the $\mathrm{TiZr}$ vessel, the Al-alloy vessel does contribute small additional Bragg peaks to the diffraction pattern. Low temperatures were achieved using a helium flow 'Orange' cryostat (AS Scientific, Abingdon, UK). The vacuum windows in the cryostat tails (usually an inner and an outer pair of foils) also contribute weak additional Bragg peaks to the diffraction patterns, and these tend to occur as doublets arising from the front and back windows. Copper collars fitted with cartridge heaters and $\mathrm{RhFe}$ temperature sensors are attached to the top and bottom of the pressure cell for accurate measurement and control of the sample temperature. Hydrostatic pressure in the system is generated and maintained using a helium gas intensifier (Stansted Fluid Power, Stansted, Essex, UK).

High-pressure investigations above $550 \mathrm{MPa}$ were made using Paris-Edinburgh ( $\mathrm{P}-\mathrm{E})$ opposed anvil presses (Besson et al., 1992) with the sample contained in encapsulated nullscattering TiZr gaskets (sample volume $=88 \mathrm{~mm}^{3}$; see Marshall \& Francis, 2002). Pressure is applied by means of an integral 250 ton $\left(1\right.$ ton $=10^{3} \mathrm{~kg}$ ) hydraulic ram, which for lowtemperature experiments is pressurized using a 5:1 mixture of $n$-pentane and isopentane. Low temperatures (at the time of these experiments) were achieved by spraying liquid nitrogen over the load frame of the $\mathrm{P}-\mathrm{E}$ cell and allowing it to pool to a depth of a few centimetres in the bottom of the sample cryotank. Warming is achieved by means of resistance heaters attached to the breech and the ram housing of the P-E cell, and temperature monitoring is performed with calibrated $\mathrm{RhFe}$ sensors mounted on the body of the $\mathrm{P}-\mathrm{E}$ cell and taped to the anvils directly adjacent to the gaskets. Pressure monitoring is achieved by use of a marker loaded with the sample. $\mathrm{Pb}$ is a suitable pressure marker since it is relatively compressible (bulk modulus $K \simeq 45 \mathrm{GPa}$ ) and unlikely to react with the sample (as opposed to sodium chloride, for example). We have tried lead powder on two occasions (experiments 5 and 7), in neither case with satisfactory results. However, a 1-2 mm-diameter pellet of polycrystalline lead punched from a foil sheet gives a good signal. The $\mathrm{Pb}$ equation of state that we used to determine the pressure was based on our own synthesis of literature values for the ambient-pressure thermal expansivity, and ultrasonic determinations of the temperature dependence of the bulk modulus, $K$, and $\partial K / \partial P$, and is given by Fortes, Wood et al. (2007). A similar literature synthesis presented by Fortes (2004) for the equation of state of ices VII and VIII was used - when these ice polymorphs were observed - to crosscheck the $\mathrm{Pb}$-derived pressures, yielding agreement within $0.01 \mathrm{GPa}$. As described below, we have had occasion to run samples in the P-E cell without the pressure marker (since the $\mathrm{Pb}$ Bragg peaks overlap some sample peaks), and we have instead used a simple linear relationship between applied load and sample pressure obtained from our first four $\mathrm{Pb}$-calibrated loadings with $\mathrm{ADH}$. Again, this has been crosschecked against the equation of state of ice polymorphs observed during an experiment. For example, during experiment 6 , the load-pressure formula yielded a pressure of $0.62 \mathrm{GPa}$, and the refined unit-cell volume of ice II in the specimen yielded a pressure of $0.56 \mathrm{GPa}$ using the experimental equation of state described in $\S 5.1 .2$.

Scattering from the tungsten carbide (WC) anvils in the Paris-Edinburgh press is reduced by coating them with cadmium foil and the use of radial collimators. Nonetheless, small parasitic peaks due to scattering from WC, and also from the nickel binder in the anvils, appear in the sample diffraction patterns.

\subsection{Sample preparation and loading}

Given the large incoherent neutron scattering cross section of the $\mathrm{H}$ atom, we used perdeuterated analogues in order to 
achieve a good signal-to-noise ratio in the measured diffraction data (cf. Finney, 1995). Deuteration is likely to have a relatively small effect on most of the properties under investigation. Bridgman's (1935) observations of the differences between $\mathrm{H}_{2} \mathrm{O}$ and $\mathrm{D}_{2} \mathrm{O}$ ices show that for a given pressure and temperature the molar volumes of $\mathrm{D}_{2} \mathrm{O}$ ice polymorphs are slightly greater than those of $\mathrm{H}_{2} \mathrm{O}$ ices; Röttger et al. (1994) determined that the unit cell of deuterated ice Ih is $0.1 \%$ larger than the protonated isotopomer. The most significant difference noted by Bridgman (1935) was in the positions of phase boundaries; in the $\mathrm{D}_{2} \mathrm{O}$ system, these were typically shifted to higher pressures and temperatures, although the magnitude of the shift exhibited no systematic trend. The largest shift was at the liquid-Ih-III triple point, for which $\left(T_{\mathrm{L}-\mathrm{I}-\mathrm{III}}^{\mathrm{D}}-T_{\mathrm{L}-\mathrm{I}-\mathrm{III}}^{\mathrm{H}}\right) / T_{\mathrm{L}-\mathrm{I}-\mathrm{III}}^{\mathrm{H}}=+1.4 \%$ and $\left(P_{\mathrm{L}-\mathrm{I}-\mathrm{III}}^{\mathrm{D}}-\right.$ $\left.P_{\mathrm{L}-\mathrm{I}-\mathrm{III}}^{\mathrm{H}}\right) / P_{\mathrm{L}-\mathrm{I}-\mathrm{III}}^{\mathrm{H}}=+6.1 \%$, where the $\mathrm{D}$ and $\mathrm{H}$ superscripts refer to deuterated and protonated species, respectively. Differences in thermoelastic properties are also small. The difference in volume thermal expansion between $\mathrm{H}_{2} \mathrm{O}$ and $\mathrm{D}_{2} \mathrm{O}$ ice Ih is insignificant (Röttger et al., 1994). Comparison of single-crystal ultrasonic measurements (Proctor, 1966; Dantl, 1968; Mitzdorf \& Helmreich, 1971) show that $\mathrm{D}_{2} \mathrm{O}$ ice Ih has an adiabatic bulk modulus, $K^{S}, \sim 1 \%$ smaller than that of $\mathrm{H}_{2} \mathrm{O}$ ice Ih, although this difference is within the measurement uncertainties.

In practice it is not possible for us to determine if a solidsolid phase transition has been shifted higher in pressure by deuteration, or if the low-pressure phase has simply persisted metastably and the transition has required some superpression to occur, as one might expect of the slower kinetics in the temperature regimes we are investigating. In the case of melting transitions, we will show that the peritectic between deuterated ammonia dihydrate and water ice is only shifted upwards by $2-3 \mathrm{~K}$ relative to the protonated isotopomer [i.e. $\left(T_{\mathrm{m}}^{\mathrm{D}}-T_{\mathrm{m}}^{\mathrm{H}}\right) / T_{\mathrm{m}}^{\mathrm{H}}=+1.1$ to $\left.+1.7 \%\right]$.

All of the experiments reported here were made on samples of nominal composition $\mathrm{ND}_{3} \cdot 2 \mathrm{D}_{2} \mathrm{O}(\mathrm{ADH})$. They were prepared by condensing $\mathrm{ND}_{3}$ gas (Aldrich Chemicals Company, 99 at \% D) into an evacuated glass bulb cooled to $\sim 220 \mathrm{~K}$ in a dry ice-acetone bath. The bulb was weighed and the contents diluted to the appropriate stoichiometry (33.3 mol\% $\mathrm{ND}_{3}$ ) with $\mathrm{D}_{2} \mathrm{O}$ (Aldrich Chemicals Company, 99 at $\%$ D). The solution was warmed, shaken and then stored in a refrigerator until use. In all studies to date, these samples have been loaded into the pressure vessels as liquids. Whilst it is straightforward to flash-freeze such samples in liquid nitrogen and then grind the resulting glass for atmospheric pressure studies, there are practical difficulties with loading the solid material into high-pressure cells and then forming a good Bridgman seal at the requisite low temperatures. $\mathrm{ND}_{3} \cdot 2 \mathrm{D}_{2} \mathrm{O}$ liquid has therefore been loaded with tufts of silica wool $(\sim 0.1 \mathrm{~g})$ to promote nucleation of a suitably random polycrystalline mass.

Loading liquid $\mathrm{ADH}$ into sample containers near room temperature usually results in a small amount of ammonia boiling out of solution; consequently, solid specimens are found to contain $5-10 \mathrm{wt} \%$ ice. This can be mitigated to some extent by loading samples in the ISIS cold room (air temperature $263 \mathrm{~K}$ ) using a syringe cooled in a dry iceacetone bath. Great care must be taken to avoid exposure of ADH liquid to $\mathrm{CO}_{2}$, which results in rapid precipitation of ammonium carbonate. Likewise, care must be exercised with regards to certain metallic alloys; for example, we have observed the formation of a blue copper-ammonia complex after using syringe needles with chrome-plated brass hubs [see Namand \& Hockberger (1992) for a similar example involving saline solution]; the most likely candidate for this complex is $\left[\mathrm{Cu}\left(\mathrm{NH}_{3}\right)_{3}\left(\mathrm{H}_{2} \mathrm{O}\right)_{2}\right](\mathrm{OH})_{2}$, known commonly as Schweizer's reagent. ADH solution decanted into a small glass bottle and dipped momentarily in liquid nitrogen yields a quite viscous material which fills nicely the encapsulated gaskets of the P-E cell.

ADH is particularly difficult to crystallize (Chan \& Giauque, 1964; Bertie \& Shehata, 1984; Yarger et al., 1993), as a result of the high viscosity of the liquid phase near the iceADH peritectic point, which is some 4000 times greater than the viscosity of water at $273 \mathrm{~K}$ (Croft et al., 1991). It is customary to flash-freeze such materials to form an amorphous solid using liquid nitrogen and then warm through the glass transition temperature. However, in ADH, devitrification is very slow, so Bertie \& Shehata (1984) proposed thermal cycling about the peritectic to promote crystallization. Because this process is time-consuming (owing to the thermal inertia of the pressure vessels), and because beamtime at national facilities is an expensive resource, we have typically done it offline prior to the start of an experiment. However, on two occasions (experiments 3 and 4 below) this process has failed offline and we have instead done it during our allocated beamtime. This has allowed us to observe the devitrification of the glass directly. Our analysis of this process is described in $\S 4.1$.

In our experience, crystallization at pressures of a few hundred MPa in the gas cell has never proven successful, even with a protracted period of temperature cycling (attempted at $550 \mathrm{MPa}$ during experiment 3 ). In the P-E cell, we normally compress the liquid under a load of $12-15$ tons $(\sim 1 \mathrm{GPa})$ at room temperature, and then cool towards a target temperature of $170-190 \mathrm{~K}$. As the sample cools and contracts, the pressure falls, dropping to $600 \mathrm{MPa}$ as the freezing point is reached near $200 \mathrm{~K}$, which typically takes several hours. Crystallization tends to occur most promptly when there is lead mixed in with the sample, but even then may require several hours of slow undercooling or super-pression to initiate. In the absence of lead it has taken over $24 \mathrm{~h}$ (experiment 6) to commence crystallization. Nevertheless, once begun, crystallization is often completed in about $30 \mathrm{~min}$.

\subsection{Data collection}

Given the extended nature of the experimental investigation reported here (31 days of beamtime at the ISIS neutron spallation source over three years) and the large quantity of data generated, we have deposited a detailed description of 
the data collection in an electronic supplement. The supplementary text ${ }^{2}$ reports full details of each individual experiment carried out, describing and illustrating (in supplementary Fig. S1) the paths followed in pressure-temperature space. A selection of relevant neutron powder diffraction data resulting from these studies are provided in supplementary Figs. S2S11.

\section{Results I: phase identification}

All of the powder indexing was carried out using DICVOLO4 (Boultif \& Louër, 2004); subsequent fitting of the powder diffraction data was performed by the method of LeBail structureless profile refinement (Le Bail et al., 1988; Le Bail, 2005) using the GSAS/EXPGUI software package (Larsen \& Von Dreele, 2000; Toby, 2001).

\subsection{ADH II}

The phase identified as ADH II was indexed first, despite having given us the greatest concerns. The appearance of $\mathrm{ADH}$ II varied quite considerably from one experiment to the next (Fig. S8), sometimes agreeing very closely with the pattern collected by Nelmes et al. (1999) and sometimes manifesting strong additional peaks. Previously (Fortes, Wood et al., 2007) we had concluded that these 'satellite peaks' were due to either (i) the specimen not being single phase; (ii) the specimen exhibiting varying degrees of preferred orientation; or (iii) super-lattice reflections. Experiment 1 afforded the sharpest reflections from this phase in which the 'satellites' were entirely absent (Fig. S2d); once we had subtracted all contributing lines from ammonia monohydrate II and ice II, the ADH II peaks were readily indexed with a high figure of merit (FOM; de Wolff, 1968; Smith \& Snyder, 1979), as cited in supplementary Table S1. Similarly, in experiment 4, we observed a minimum of parasitic peaks, and were able to follow these through the ADH I-II phase transition, thus eliminating both accessory ice IX [see the supplementary material and Bauer et al. (2008) regarding the presence of this phase] and residual ADH I (Fig. S3); these patterns were also indexed with the same monoclinic cell and an excellent FOM. One possible corollary to confident indexing is the observation of sensible absences, a systematic pattern of zero-intensity Bragg reflections which indicate the presence of certain symmetry elements in the unit cell and may identify the space group of the crystal. In this case, the absences (all $h 0 l$ with $h+l$ odd) identify a primitive monoclinic cell with an $n$-glide parallel to (010), narrowing the likely space groups to $P n, P 2 / n$ or $P 2_{1} / n$.

We considered initially the possibility that all of the 'satellite' peaks observed in experiments 2 and 3 (Figs. S $a$ and S $8 b$ ) were superstructure reflections. However, no supercell of the ADH II cell could be identified that accounted for these additional Bragg peaks. An attempt made to index these peaks

\footnotetext{
2 The supplementary text, figures and tables discussed in this paper are available from the IUCr electronic archives (Reference: KS5224). Services for accessing these data are described at the back of the journal.
}

using the experiment 2 data set gave the rather surprising result that a monoclinic unit cell remarkably similar to that of ADH II was identified with a very large FOM (Table S1). Subsequent profile refinement established that the volume of this cell was almost identical (although not quite within $3 \sigma$ ) to the original $\mathrm{ADH}$ II unit cell, with very small strains, $\Delta c / c=$ $-1.8 \%, \Delta b / b=+0.8 \%$ and (for a monoclinic crystal, the direction orthogonal to the $b c$ plane $) \Delta(a \sin \beta) /(a \sin \beta)=$ $+0.9 \%$, with respect to ADH II. Given the similarity of the two cells, we conclude that these two crystals have the same heavyatom structure, and the difference between them is probably due to a difference in the directionality of the hydrogen-bond ordering scheme; as a result we will call these phases ADH II $a$ and $\mathrm{ADH} \mathrm{II} b$. A similar incommensurate composite structure is observed in the high-temperature para-electric phase of $\left(\mathrm{NH}_{4}\right)_{2} \mathrm{SO}_{4}$, where two orthorhombic unit cells with slightly different dimensions have been found to co-exist (Shmyt'ko et $a l ., 2002)$. We have observed that ADH II $b$ typically forms only when the ADH I $\rightarrow$ II phase boundary is crossed rapidly: in experiments 2 and 3 the pressure was increased directly to $550 \mathrm{MPa}$ in a matter of minutes, whereas in experiment 4 we stepped through the transition over the course of $\sim 30 \mathrm{~min}$, and in experiment 1 we grew ADH II from the liquid phase over many hours. Thus ADH II $b$ may represent some structurally frustrated metastable state that occurs when the I $\rightarrow$ II transition takes place very quickly.

\section{2. АMH II}

Fortes, Wood, Knight et al. (2003) and Fortes (2004) wrote of experiment 1 that 'analysis of the data from the highpressure experiment indicates that the new pattern represents a complex mixture of both $\mathrm{ADH}$ II, phase II of ammonia monohydrate (AMH II), ice IX and possibly ice II'. Although we later erroneously identified the AMH II + ice II mixture as a single phase dubbed ADH III, a more thorough analysis of the data from experiment 4 shows that our former conclusion was correct. Supplementary Fig. S9 compares our results from experiments 2 and 4 with those of Nelmes \& Loveday (1998) and with the simulated neutron powder diffraction pattern of ice II. That this pattern is due to a mixture of AMH II and ice II is confirmed by the observation of partial melting (Fig. S5). When the Bragg peaks of ice II are accounted for, those peaks remaining due exclusively to the high-pressure monohydrate are indexed with a high-FOM orthorhombic unit cell (Table S1). Comparison with the AMH I compression data of Loveday \& Nelmes (2004) - see $\$ 5.3 .1$ below - shows that this indexing is consistent with 16 formula units per unit cell and hence considerable structural complexity. The systematic absences point to a primitive cell with an $a$-glide parallel to (001) ( $h k 0$ absent with $h=$ odd) and a $c$-glide parallel to (010) ( $h 0 l$ absent with $l=$ odd). However, ambiguity remains concerning the symmetry element parallel to (100) since the requisite reflections may be simply not detected above the noise rather than absent altogether; the balance of minimizing additional reflections (i.e. 011 and 021 are not obviously present) suggests a $c$-glide parallel to (100) $(0 \mathrm{kl}$ absent with $l=$ 
odd), yielding the space group $P c c a$, although $P n c a$ and $P b c a$ are not excluded. Since this paper was submitted for publication, the structure of AMH II has been solved by one of us (Fortes) in space group Pbca, and that work is reported elsewhere.

Having indexed ADH II $a$ and AMH II, and having clearly identified the accessory ice phases in the experiment 1 postmelting data (supplementary Fig. S2), we were able to refine the unit-cell parameters of both ADH II $a$ and AMH II to very high precision (roughly one part in $10^{5}$, comparable to our best single-phase powder data collected in other high-pressure experiments with HRPD). The results of this Le Bail structureless profile refinement are shown in supplementary Fig. S10 and tabulated in Table S2. Owing to the very high quality of these data (sharp, well resolved peaks and excellent signal-to-noise ratio), these are the most precisely determined unit-cell parameters of any of our high-pressure phases reported here.

\subsection{ADH IV}

Over the course of many experimental runs, both with and without a $\mathrm{Pb}$ pressure marker, we have observed ADH IV coexisting with ice II, ice VI and AMH II. In none of these runs have we observed a pure ADH IV pattern; our only ice-free data were collected from a sample containing the $\mathrm{Pb}$ pressure marker. Hence, identification of the reflections due solely to ADH IV has required careful cross referencing of numerous data sets. Although the orthorhombic indexing obtained from this edited data set of reflections did not have a very high FOM (Table S1), and also had a large zero-shift, the relatively high symmetry, the acceptable molar volume (consistent with eight molecules per unit cell for a sensible volume difference between ADH IV and ADH II) and the observation of plausible systematic absences suggested that this solution was likely to be correct. Subsequent LeBail profile refinements of a number of different data sets with this orthorhombic unit cell generally support this conclusion; Fig. S11 is an example of such a refinement using one of our best data sets for ADH IV (in this instance co-existing with ice II), the powder statistics and unit-cell parameters being reported in Table S3.

The systematic absences indicate a primitive unit cell with an $n$-glide parallel to (001) ( $h k 0$ absent for $h+k=$ odd) and a $c$-glide parallel to (010) ( $h 0 l$ absent with $l=$ odd). The symmetry element parallel to (100) is ambiguous; the pertinent reflection, 021 , is very close to 121 , and it is not altogether clear at the resolution of the PEARL/HiPr powder data if 021 is absent. If 021 is a genuine systematic absence then this satisfies either $0 k l$ with $l=$ odd, giving a $c$-glide parallel to (100), or $0 k l$ with $k+l=$ odd, which would give an $n$-glide parallel to (100). Hence, we cannot entirely rule out space groups $P m c n, P b c n$ or $P 2_{1} c n$, but the most likely candidates are Pccn or Pncn. It is worth observing that Pmcn is equivalent to Pnma, the second most common space group in which inorganic compounds crystallize (Mighell et al., 1977).

Boone (1989) published images of ADH crystals grown from the liquid phase in a diamond anvil cell at a pressure of $\sim 700 \mathrm{MPa}$. Boone attempted to obtain X-ray diffraction data from these single crystals but was not successful (S. C. Boone, personal communication); if we hypothesize that these are ADH IV crystals, then we can assess whether or not our unitcell indexing is consistent with the observed growth morphology and interfacial angles. In Fig. 1 we have reproduced two of the images published by Boone \& Nicol (1991), alongside model crystals constructed using WinXMorph (Kaminsky, 2005) with our ADH IV unit-cell indexing and the assumption that the families of faces expressed have the smallest Miller indices. In Fig. 1(c) it appears that the crystal is growing with the (010) face parallel to the diamond culet, thus affording us the most favourable geometry to measure selected interfacial angles from the image. Those labelled have the values $\alpha=126.5^{\circ}, \beta=116^{\circ}$ and $\gamma=154^{\circ}$. The same angles on the calculated crystal model have the values $\alpha=(111) \wedge$ $(\overline{1} \overline{1} \overline{1})=126.185^{\circ}, \beta=(111) \wedge(110)=116.907^{\circ}$ and $\gamma=(001) \wedge$ $(101)=153.093^{\circ}$. There is sufficient agreement between the observed and predicted morphologies to conclude that axial ratios of ADH IV are consistent with the interfacial angles of the crystals photographed by Boone \& Nicol (1991).

\section{Results II: crystallization kinetics}

Although we had not intentionally set out to measure the rates of crystal growth in the ammonia-water system, we have been able to make observations that provide constraints on the growth kinetics. These afford a valuable comparison with similar data in the pure water system, and are also of use in understanding the crystallization behaviour of cryolava flows
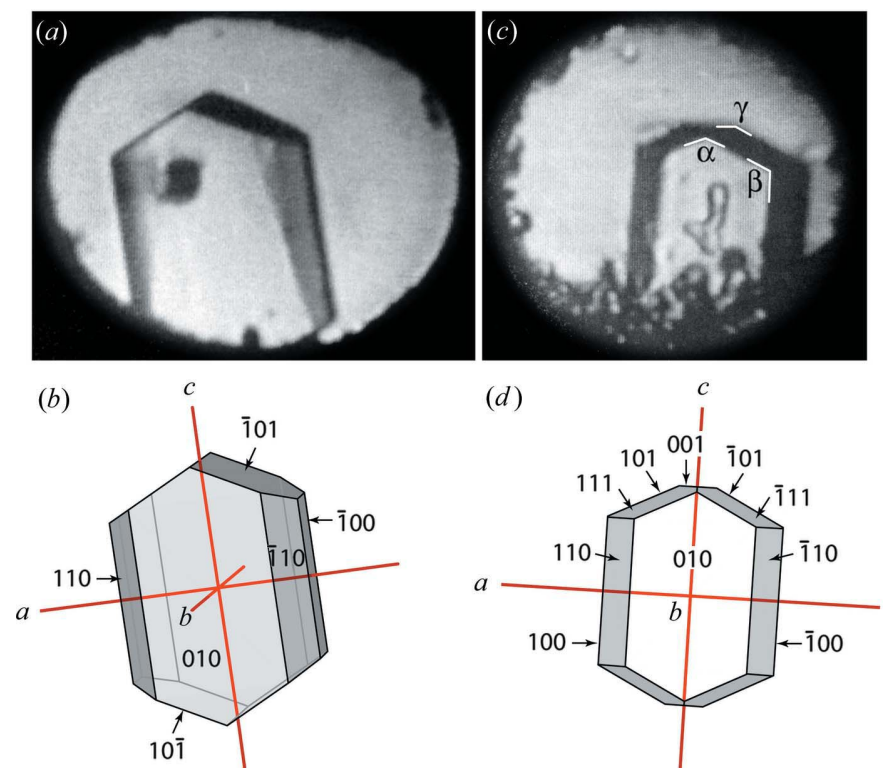

Figure 1

(a) and (c) Optical micrographs of ADH crystals grown from solution in a diamond anvil cell, reproduced from Figs. $2(b)$ and 5 of Boone \& Nicol (1991) with the permission of the Lunar and Planetary Institute. (b) and (d) Model crystals, drawn using the program WinXMorph (Kaminsky, 2005) using the orthorhombic unit-cell indexing of ADH IV reported in Table S1; these have been oriented to best match the crystals in $(a)$ and $(c)$. 
on icy planetary bodies. The release of latent heat into a terrestrial silicate lava flow has a significant impact on the flow's heat balance (e.g. Settle, 1979) and may affect the overall dimensions of the flow; similar recalescence in a cryolava flow, as a possibly amorphous crust undergoes devitrification, should be accounted for in models that seek to relate flow morphology with flow composition.

\subsection{Crystallization of $\mathrm{ADH} I$ at $15 \mathrm{MPa}$}

We observed the devitrification of amorphous ammonia dihydrate under $15 \mathrm{MPa}$ of He gas pressure on two occasions which have allowed us to understand the kinetics of the process and to constrain the peritectic melting point of the
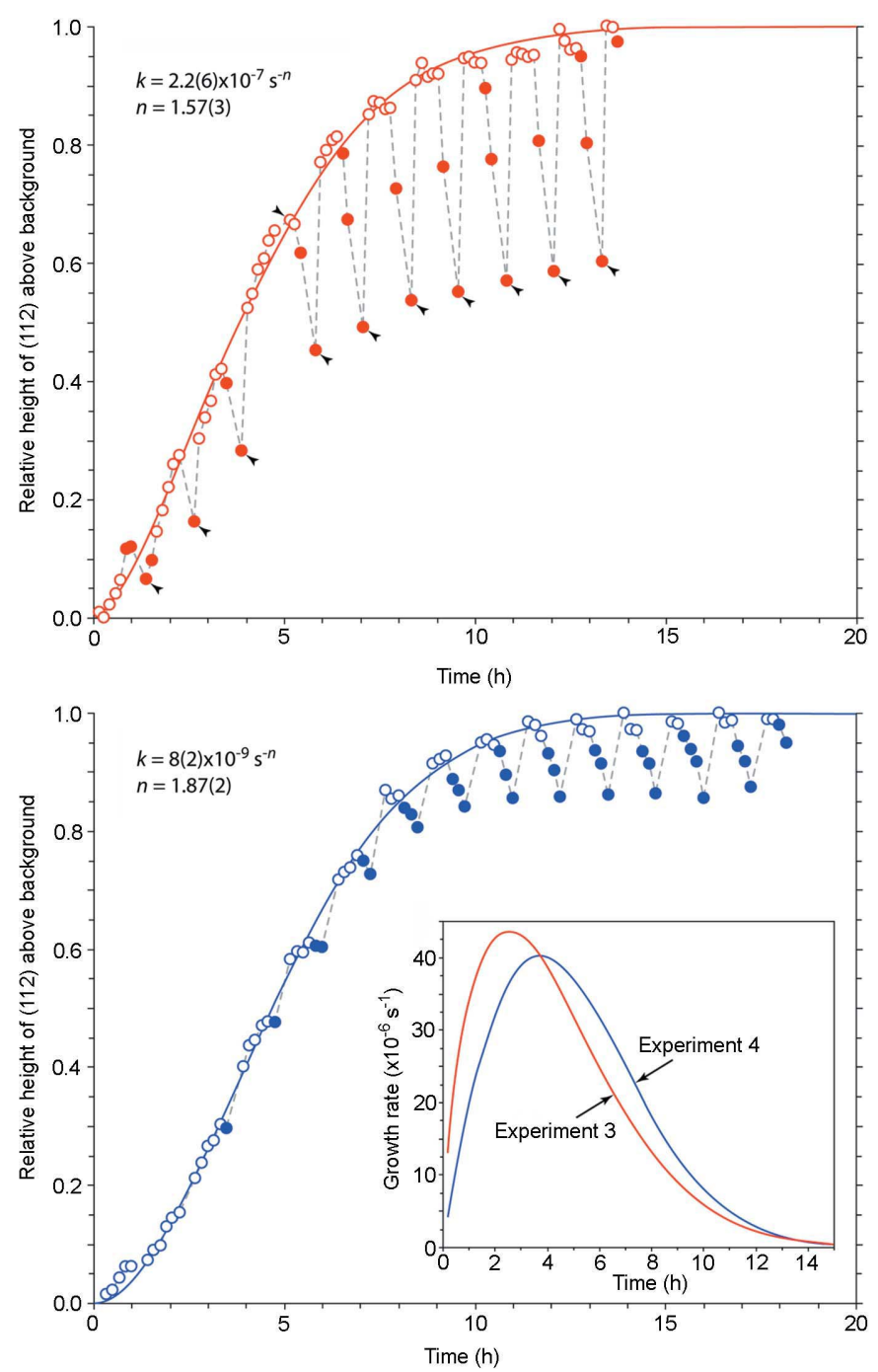

Figure 2

Plots of the relative ADH I (112) Bragg peak height above background as a function of time, collected during temperature cycling in $(a)$ experiment 3 and $(b)$ experiment 4 . Data indicated with open circles were fitted to equation (1), and the parameters obtained are indicated. Filled circles were not used in the fitting. In $(a)$, points marked with a black arrow represent data collected at a nominal temperature of $173 \mathrm{~K}$. Note that cycle 4 was interrupted midway through and the temperature reset to $173 \mathrm{~K}$. In (b) the inset shows the growth rates as a function of time determined from the KJMA equation parameters. deuterated species. Fig. 2 shows a very simple measure of the progress of ADH I crystallization, the relative height of the strong 112 peak of ADH phase I $(d$ spacing $=2.9131 \AA$ near atmospheric pressure) as a function of time. In experiment 3 (Fig. $2 a$ ) we warmed from 173 to $179 \mathrm{~K}$ in $1 \mathrm{~h}$ and cooled back to $173 \mathrm{~K}$ in $15 \mathrm{~min}$, repeating this cycle 12 times. The thermal phase lag $(\sim 10 \mathrm{~min})$ in the response of the sample relative to the temperature recorded by the sensor is revealed by the drop in the 112 peak intensity at $178-179 \mathrm{~K}$, with the minimum at $173 \mathrm{~K}$ in the next thermal cycle (arrowed). These dips in the 112 peak height are a manifestation of the partial melting at $178-179 \mathrm{~K}$, which is only $2-3 \mathrm{~K}$ higher than the peritectic temperature of protonated $\mathrm{ADH}[176.09$ (5) K] measured by Chan \& Giauque (1964); hence $\left(T_{\mathrm{m}}^{\mathrm{D}}-T_{\mathrm{m}}^{\mathrm{H}}\right) / T_{\mathrm{m}}^{\mathrm{H}} \simeq+1.1$ to $+1.7 \%$, similar to the values obtained by comparison of the $\mathrm{H}_{2} \mathrm{O}$ and $\mathrm{D}_{2} \mathrm{O}$ phase relations by Bridgman (1935).

In our second online annealing (experiment 4, Fig. $2 b$ ) we wished to avoid the onset of partial melting, since we believed this slowed the annealing process. The temperature was cycled from 173 to $178 \mathrm{~K}$ over a period of $1 \mathrm{~h}$ and cooled back to $173 \mathrm{~K}$ in $15 \mathrm{~min}$, repeating the cycle 15 times; clearly, some melting still occurred, but to a lesser degree than in the previous experiment.

Despite neither experiment being strictly isothermal, we can nevertheless fit the well known Kolmogorov-JohnsonMehl-Avrami (KJMA) expression (Kolmogorov, 1937; Johnson \& Mehl, 1939; Avrami, 1939, 1940) to some of these data:

$$
X=1-\exp \left(-k t^{n}\right),
$$

where, in this instance, $X$ is the relative height of the 112 peak, a proxy for the proportion of the specimen that has crystallized, $k$ is a rate constant (units time ${ }^{-n}$ ), and $n$ is the 'Avrami constant', which contains information on the time dependence of nucleation, the geometry of the growing crystallites and the nature of the reaction process (interfacial versus diffusional). The Avrami constant $n$ is the sum of two components, $\alpha+\beta$. The number density of nucleation centres $(N)$ has a model time dependence of the form $N \propto t^{\alpha}$, such that for $\alpha=0$, all nucleation sites are present at $t=0$ (the growth medium is nuclei site saturated), and for $\alpha=1$, the nucleation rate is a constant: for $\alpha<1$, the nucleation rate slows with time, and for $\alpha>1$, the nucleation rate increases with time. The term $\beta$ expresses the dimensionality of the growth geometry (i.e. onedimensional $=$ needle-like or acicular growth, twodimensional $=$ platy or tabular growth, three-dimensional = blocky or globular growth) and has the values 1, 2 or 3 for one-, two- or three-dimensional growth, respectively, when the reaction process is interfacial, and has the values $0.5,1$ or 1.5 when the reaction process is diffusional. One can construct a table showing a range of possible values of $n$ which may be used to help understand the process under observation (Table S4), although clearly there are some ambiguities unless independent data on crystal growth morphology, for example, are at hand. For our purposes, we can infer to a certain extent the nature of the reaction process, and also use the crystallographic unit cell to estimate the likely growth morphology. 
The data in Fig. 2(a) are fitted with equation (1) to obtain $k=2.2(6) \times 10^{-7} \mathrm{~s}^{-n}$ and $n=1.57$ (3); the data in Fig. 2(b) yield $k=8(2) \times 10^{-9} \mathrm{~s}^{-n}$ and $n=1.87$ (2). From these, we can also obtain the relative growth rates as a function of time, $\mathrm{d} X /$ $\mathrm{d} t$ [equation (2)], the peak growth rate, $t_{\max }$ [equation (3)], and the half-life of the growth process, $t_{1 / 2}$ [equation (4)]:

$$
\begin{aligned}
\mathrm{d} X / \mathrm{d} t & =k n t^{n-1} \exp \left(-k t^{n}\right), \\
t_{\max } & =[(n-1) / k n]^{1 / n}, \\
t_{1 / 2} & =-\ln (1 / 2) / k^{1 / n} .
\end{aligned}
$$

The growth rates $\mathrm{d} X / \mathrm{d} t$ are plotted in the inset to Fig. 2(b). In the first annealing (experiment 3 ), the peak growth rate is $4.4(11) \times 10^{-5} \mathrm{~s}^{-1}$, which occurs after 2.5 (6) $\mathrm{h}$, and the halflife is $3.3(8) \mathrm{h}$. In the second annealing (experiment 4), the peak growth rate is $4.0(6) \times 10^{-5} \mathrm{~s}^{-1}$, which occurs after 3.8 (4) h, and the half-life is $4.0(5) \mathrm{h}$.

The values of $n$ are very similar to those found when both $\mathrm{H}_{2} \mathrm{O}(n=1.07-1.74)$ and $\mathrm{D}_{2} \mathrm{O}(n=1.01-1.17)$ ice crystals are grown in a matrix of amorphous solid water at temperatures of 138-155 K (Hage et al., 1994, 1995). $\mathrm{D}_{2} \mathrm{O}$ ice has been found to crystallize more slowly, and later, than $\mathrm{H}_{2} \mathrm{O}$ ice at the same temperature. The maximum growth rates we obtain are 1.4 orders of magnitude less than those measured by Hage et al. (1994, 1995), which might be due to differences in diffusion rates, or more likely to the very small degree of undercooling in our experiment (0-6 K) compared with their study $(>120 \mathrm{~K})$.

We would expect growth from an amorphous solid to be diffusion limited, and we would also expect, given the cubic symmetry of ADH I, that the growth geometry must be threedimensional, which gives $\beta=1.5$. We attribute the difference in $n$ between the two observations to differences in nucleation rate. Given the similarity in temperatures we might anticipate that these would be the same; however, new nuclei may be being destroyed by the partial melting occurring in experiment 3 , yielding a smaller value of $\alpha$. The observed values of $n$ are therefore plausibly consistent with $\alpha \simeq 0.1$ in experiment 3 and $\alpha \simeq 0.4$ in experiment 4 . Interestingly, despite the lower nucleation rate, the overall growth rates in experiment 3 are marginally greater, despite the cyclical partial melting.

\subsection{Crystallization at high pressures}

Having indexed all of the high-pressure phases observed in experiment 1 , we have been able to make a similar analysis of their crystal growth kinetics. For each phase we sought to identify strong reflections with no overlap and a clear region of adjacent background, so as to obtain reliable measurements of the peak height as a function of time. For ice II we selected the 122 reflection at $2.4923 \AA$; for ADH II $a$ we chose the 121 peak at $2.8311 \AA$; for AMH II we chose the 610 reflection at $2.8649 \AA$ : these three peaks are indicated in supplementary Fig. S2. The curves measured from these peaks are shown in Fig. 3, and each is fitted with the KJMA equation [equation (1)].
The crystallization behaviour of each phase is strikingly different. Growth of ice II, which is the first phase to appear (being present in the pattern measured at $t=9 \mathrm{~min}$ after cooling back to $174 \mathrm{~K}$; Fig. S2a) is fitted by a KJMA equation with $k=1.5(9) \times 10^{-4} \mathrm{~s}^{-n}$ and $n=1.04$ (7). Both ADH II and AMH II are clearly present in the data measured at $t=45 \mathrm{~min}$ (Fig. S2 $b$ ) but not $t=9 \mathrm{~min}$; for ADH II $a$ the KJMA parameters are $k=5(3) \times 10^{-8} \mathrm{~s}^{-n}$ and $n=1.87$ (8), and for AMH II they are $k=1.5(10) \times 10^{-6} \mathrm{~s}^{-n}$ and $n=1.43(7)$. Growth rates are plotted in the inset to Fig. 3(d). Ice II grows at a maximum rate of $1.9 \times 10^{-4} \mathrm{~s}^{-1}$ at $t=3.5 \mathrm{~min}$, and has a growth half-life of just $55 \mathrm{~min}$, whereas the two ammonia hydrates exhibit rather more sedate growth; ADH II reaches a maximum growth rate of $1.0 \times 10^{-4} \mathrm{~s}^{-1}$ at $t=1.5 \mathrm{~h}$ (half-life $=$ $1.6 \mathrm{~h}$ ), and AMH II reaches a maximum growth rate of $6.4 \times$ $10^{-5} \mathrm{~s}^{-1}$ at $t=1.4 \mathrm{~h}$ (half-life $=2.2 \mathrm{~h}$ ). ${ }^{3}$ The decline in growth rate is slowest in AMH II, and this phase is still measurably growing $5 \mathrm{~h}$ after $t=0$, whereas ice II has essentially completed its growth in half that time.

Ice II is almost certainly growing directly from the liquid phase, and the reaction is likely to be interfacial, nucleating upon either the silica wool or pre-existing crystals of ice IX $(\alpha=0)$; the observed value of $n(\sim 1.0)$ is therefore suggestive of one-dimensional growth of needle-like crystals. The unitcell symmetry, and the crystal structure, are consistent with the growth of acicular crystals extended along the threefold axis. Only a single observation of ice II crystals exists in the literature (see Fig. 3 of Grasset et al., 2005), but this nonetheless confirms our hypothesis that the growth habit is acicular.

Both ADH II $a$ and AMH II are likely to be growing in a highly viscous under-cooled liquid, or an amorphous solid, and so the reaction is probably diffusion limited. Given the unitcell dimensions, $\mathrm{ADH}$ II $a$ is most likely to exhibit near isotropic three-dimensional growth $(\beta=1.5)$, whereas the unit cell of AMH II lends itself to the growth of tabular crystals (two-dimensional growth, $\beta=1.0$ ), the largest faces having the Miller indices (100). If these assumptions are correct, then the Avrami constants for both phases are consistent with very similar nucleation rates, $\alpha \simeq 0.4$, which is effectively identical to that inferred from the ADH I growth measurements.

Both the sequence of crystallization and the magnitude of the growth rates can be understood in a qualitative sense by reference to the sketch phase diagram in Fig. 4, which has phase boundaries essentially identical to those observed in this system at atmospheric pressure. Having caused partial melting of a mixture of ADH I + ice IX, followed immediately by quenching back to $174 \mathrm{~K}$, the first phase to crystallize is the liquidus phase, ice II, with the high growth rate dictated by the large degree of under-cooling (probably $\sim 20 \mathrm{~K}$ ). Crystallization of significant ice II drives the composition of the residual liquid towards a more ammonia-rich concentration, probably along a metastable extension of the ice II liquidus. The quenched residue now finds itself far into the ADH II +

\footnotetext{
${ }^{3}$ Note that we do not give errors on $t_{\max }, t_{1 / 2}$ or $\mathrm{d} X / \mathrm{d} t$ here; the relative propagated uncertainty is up to $85 \%$ on some of these derived quantities.
} 
AMH II phase field (thus, these commence crystallizing together), with the former experiencing a greater degree of under-cooling, and hence a larger maximum growth rate, than the latter.

Although these experiments were not designed to obtain growth kinetics data, useful inferences are nonetheless possible, and indeed recommend to us that proper kinetic measurements should be made, possibly using time-resolved neutron diffraction.

\section{Results III: densities as a function of pressure and/or temperature}

\subsection{Ice polymorphs}

The densities reported below are summarized in Figs. 5 and 6 . We compare our results with literature data on the equations of state (either experimental or computational), in part to test those equations of state, and in part to confirm that our pressure calibrations are accurate.

The use of helium in high-pressure studies of water ice is known to be problematic, since $\mathrm{He}$ is able to penetrate the large hexagonal ring structures in ice Ih and ice II, effectively
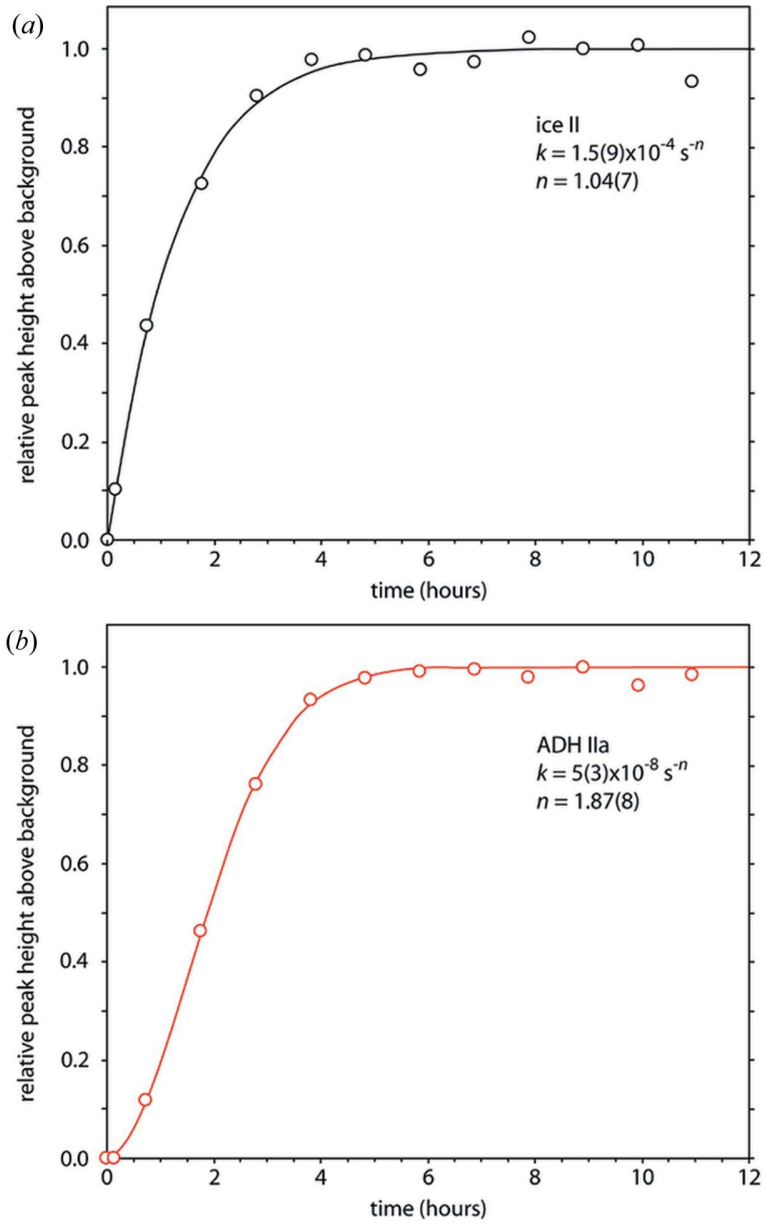

dissolving in the solid ice to form a helium hydrate (Kahane et al., 1969; Arnold et al., 1971; Londono et al., 1992). The consequence of this is both a change in the incompressibility of ice II (it becomes much stiffer; Lobban et al., 2002) and a total suppression of ice phases III and V; ice II-structured $\mathrm{He}$ hydrate becomes the liquidus phase at pressures of 250 $850 \mathrm{MPa}$ (e.g. Dyadin et al., 1999). In our previous work on ice II, we overcame this problem by using argon as the pressuretransmitting medium, although the comparatively high boiling point with respect to helium poses its own problems (Fortes et $a l ., 2005)$. In our experiments on the ammonia hydrates, we were satisfied that helium cannot penetrate the low-pressure structures since these lack the large open cavities found in ices Ih and II, and that it does not in fact do so, since we find quantitative agreement in the phase behaviour observed by us under helium and by others using different pressure media (e.g. Hogenboom et al., 1997). The question remains as to whether the ice polymorphs reported in this work, typically present as accessory phases encased in a solid matrix of ammonia hydrate, can be infiltrated by helium. Firstly, the presence of ice IX (the proton ordered form of ice III - see below) suggests to us that helium is not penetrating the accessory ice phase, or else this phase would likely not occur.
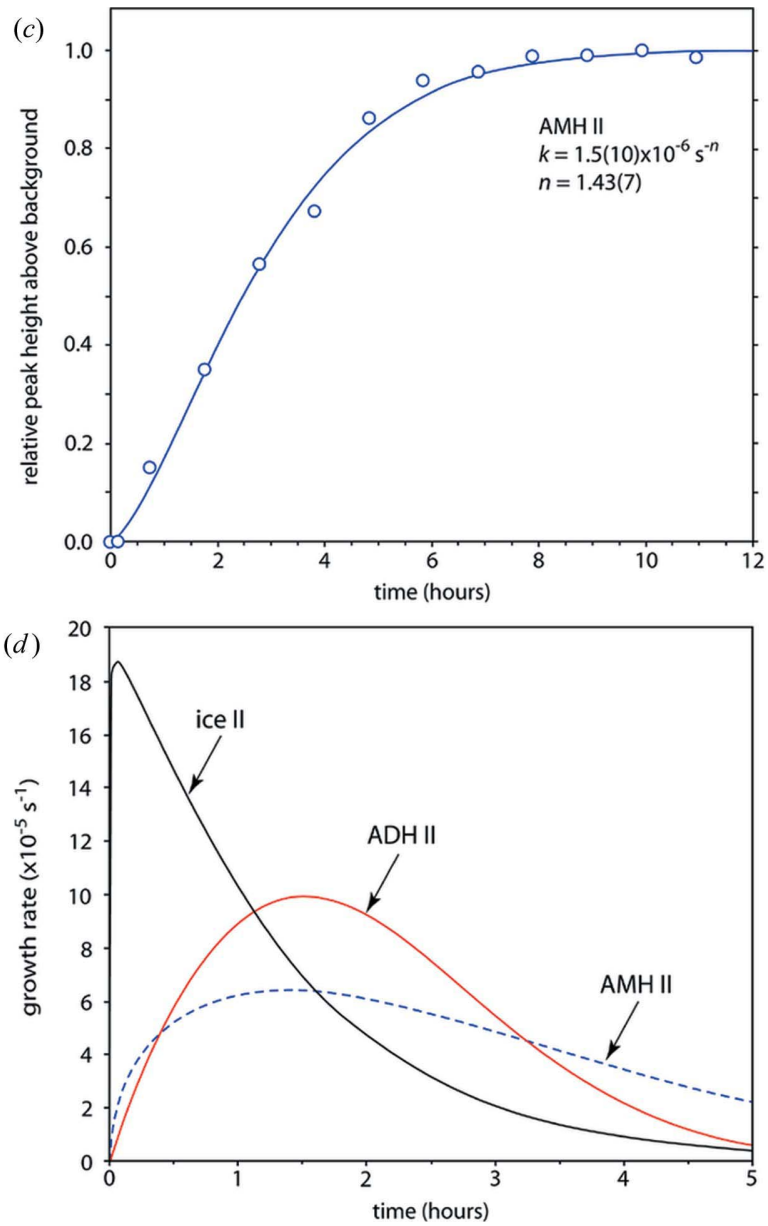

Figure 3

Plots of relative Bragg peak height above background as a function of time for ice II $(a)$, ADH II $a(b)$ and AMH II $(c)$, collected during crystallization at $174 \mathrm{~K}, 443 \mathrm{MPa}$ in experiment 1 (see text for details of the reflections used, and Fig. S2). The data were fitted to equation (1), and the parameters obtained are indicated. $(d)$ The growth rates as a function of time determined from the KJMA parameters. 
Neither do we see any convincing evidence in the unit-cell dimensions of the ice phases for infiltration of helium; the pressure dependence of the ice Ih and ice IX unit-cell volumes agrees with that predicted from their elastic constants, and only the anomaly in the ice II $c / a$ ratio at $550 \mathrm{MPa}$ cannot uniquely be attributed to anisotropic stress or helium penetration. We therefore conclude that He probably does not diffuse through the ammonia hydrate matrix and dissolve in the accessory ice on the timescale of our laboratory studies. These points are discussed further in the following sections.

5.1.1. Ice Ih. Ice Ih was observed as an accessory phase $(\sim 12.5 \mathrm{wt} \%)$ with ADH I in experiment 1 . Unit-cell parameters for ice Ih were refined using the Rietveld method at six pressure points along the $175 \mathrm{~K}$ isotherm, between 41.1 and 300.8 MPa. An isothermal Murnaghan integrated linear equation of state (MILEOS; Murnaghan, 1944) was fitted to the unit-cell volumes:

$$
V_{0} / V_{P}=\left(1+P K_{0}^{\prime} / K_{0}\right)^{1 / K_{0}^{\prime}}
$$

where $V_{0}$ is the molar volume at $P=0, V_{P}$ is the molar volume at pressure $P, K_{0}$ is the isothermal bulk modulus (= incompressibility) at $P=0$, and $K_{0}^{\prime}$ is the first pressure derivative of the bulk modulus, $(\partial K / \partial P)_{T}$ evaluated at $P=0$. Since $V, K$ and $K^{\prime}$ are all temperature dependent to some extent, we cite these parameters with subscripts $P, T$.

A least-squares fit of equation (5) to our ice Ih refined unitcell volumes gave the parameters $V_{0,175}=129.02(6) \AA^{3}$,

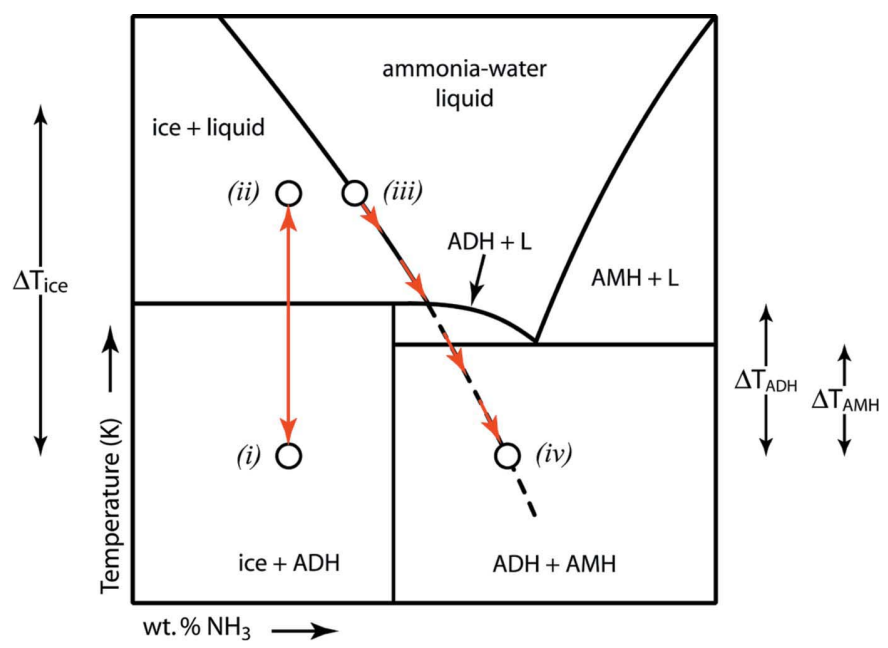

Figure 4

A schematic $T-X$ slice through the $P-T-X$ phase diagram at $\sim 450 \mathrm{MPa}$, indicating the sequence of events that probably occurred in experiment 1 after melting and subsequent cooling. The specimen of ADH + ice IX was warmed from $174 \mathrm{~K}(i)$ to $179 \mathrm{~K}(\mathrm{ii})$, resulting in partial melting to ice + liquid. The composition of the liquid co-existing with the ice phase is indicated by (iii). On cooling back to $174 \mathrm{~K}$, the liquid crystallizes the stable ice phase under these $P, T$ conditions (ice II) and the composition evolves down the liquidus towards (iv). At the end of the metastable extension of the ice II liquidus (dashed black line), the residual supercooled liquid has a composition far into the $\mathrm{ADH}+\mathrm{AMH}$ stability field, co-crystallizing high-pressure phases of each compound. The approximate degree of undercooling for each phase is marked, being greatest for ice II and least for AMH II; the maximum growth rates appear to be proportional to the degree of undercooling.
$K_{0,175}=9.7$ (6) $\mathrm{GPa}$ and $K_{0,175}^{\prime}=6$ (4). The unit-cell volume is in excellent agreement with that obtained from powdered $\mathrm{D}_{2} \mathrm{O}$ ice Ih by Röttger et al. (1994) [128.99 (1) $\left.\AA^{3}\right]$ and from powdered $\mathrm{D}_{2} \mathrm{O}$ ice $\mathrm{Ih}$ in a matrix of $\mathrm{MgSO}_{4} \cdot 11 \mathrm{D}_{2} \mathrm{O}$

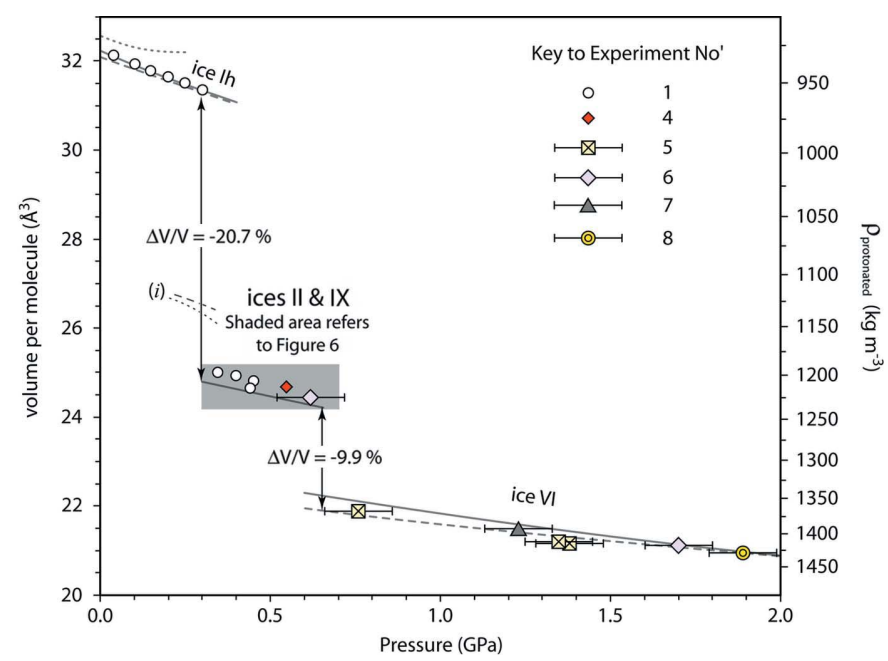

Figure 5

Molecular volumes (left axis) and densities (right axis) of the various ice polymorphs observed during our studies. Solid lines are empirical equations of state of our own derivation for ices II $(175 \mathrm{~K})$ and VI (190 K), and of Feistel \& Wagner's (2006) for ice Ih (175 K). Dashed lines show the theoretical equation of state from Noya et al. (2007). Dotted lines are the equations of state from Choukroun \& Grasset (2007). The two lines marked $(i)$ are the ice III equations of state from Noya et al. (2007) and Choukroun \& Grasset (2007) evaluated at 175 and $180 \mathrm{~K}$, respectively. The region covered by the grey box is expanded upon in Fig. 6.

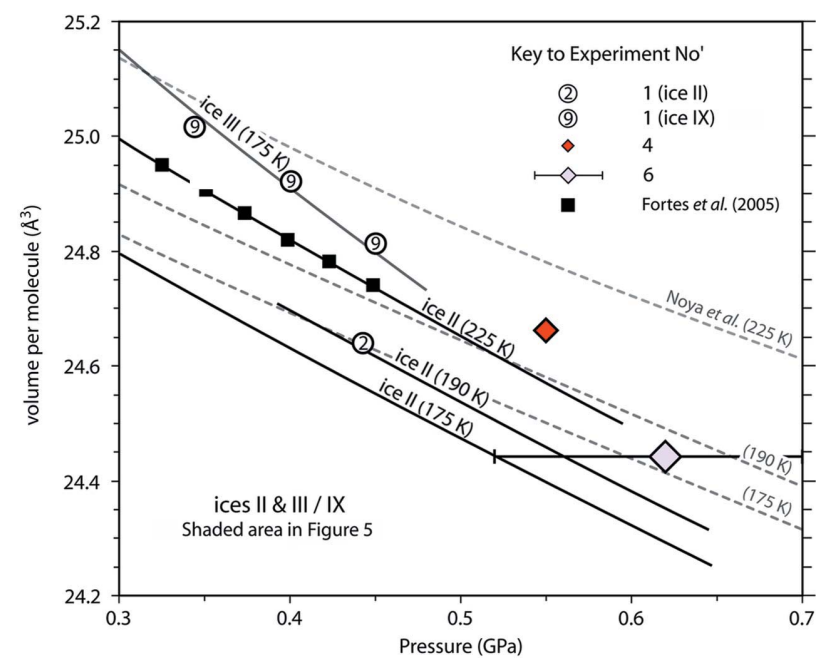

Figure 6

Molecular volumes of ices II and IX, expanded from Fig. 5 in order to illustrate the misfits between our observations and empirically derived and theoretically derived equations of state. Note that the open circles representing experiment 1 have been labelled to distinguish ice II (marked with a ' 2 ') and ice IX (marked with a ' 9 '). Solid black squares are the ice II data of Fortes et al. (2005) collected in an Al 7075 pressure cell, pressurized under argon gas, along the $225 \mathrm{~K}$ isotherm. The solid black line marked 'ice II $(225 \mathrm{~K})$ ' is fitted to the latter data set. The derivation of the other solid lines is described in the text. The dashed lines represent the theoretical equation of state of Noya et al. (2007) evaluated at the indicated temperatures. 
[129.08 (1) $\AA^{3}$; Fortes et al., 2008], both at $175 \mathrm{~K}$. The isothermal bulk modulus of $\mathrm{D}_{2} \mathrm{O}$ ice $\mathrm{Ih}$ is determined from the relationship $K^{T}=K^{S} /(1+\alpha \gamma T)$, where $K^{T}$ and $K^{S}$ are the isothermal and adiabatic bulk moduli, respectively, $\alpha$ is the volume thermal expansion coefficient, and $\gamma$ is the Grüneisen parameter. At $175 \mathrm{~K}$, Röttger et al. (1994) give $\alpha=107.8 \times$ $10^{-6} \mathrm{~K}^{-1}$ and $\gamma=0.70 ; K^{S}$ is calculated from the elastic constants measured by Mitzdorf \& Helmreich (1971) $\left[K_{0,175}^{S}=\right.$ 9.5 (5) $\left.\mathrm{GPa}^{4}\right]$. Hence, $K_{0,175}^{T}=9.3(5) \mathrm{GPa}$. The pressure dependence of the bulk modulus has been measured at $77 \mathrm{~K}$ over the range $0-1 \mathrm{GPa}$ using ultrasonic techniques by Gromnitskaya et al. (2001); we have fitted a simple linear expression to their data to obtain $K_{0,77}^{\prime}=7.0$ (1). If we repeat our least-squares fit of the MILEOS with $K_{0,175}^{\prime}$ fixed at 7.0, we find $V_{0,175}=129.03(3) \AA^{3}$ and $K_{0,175}=9.6(1) \mathrm{GPa}$. In Fig. 5 our data and fitted equation of state are compared with the molecular volumes calculated from the empirically derived equations of state of Feistel \& Wagner (2006) and Choukroun $\&$ Grasset (2007), and the theoretical equation of state of Noya et al. (2007) derived from interatomic potential calculations. Feistel \& Wagner's equation of state, evaluated at $175 \mathrm{~K}$ and re-parameterized in terms of equation (5), has $V_{0,175}=128.862 \AA^{3}, K_{0,175}=9.78 \mathrm{GPa}$ and $K_{0,175}^{\prime}=7.1$, which is in very close agreement with our fitted MILEOS and allows us to conclude that the accessory ice Ih observed by us was not affected by the presence of helium that may have diffused through the surrounding ADH I crystals; the difference in molecular volume at atmospheric pressure amounts to $0.12 \%$, becoming smaller with pressure, and being zero at an extrapolated pressure of $0.68 \mathrm{GPa}$. The agreement with the empirical parameterization of Choukroun \& Grasset (2007) is much poorer; their equation of state (evaluated at their lowest recommended temperature, $180 \mathrm{~K}$ ) overestimates the zeropressure molecular volume by $0.94 \%$; whilst their zero-pressure bulk modulus $\left(K_{0,180}=9.66 \mathrm{GPa}\right)$ is in excellent agreement with our results and with Feistel \& Wagner (2006), the first pressure derivative is too large by an order of magnitude $\left(K_{0,180}^{\prime}=70\right)$, leading to the anomalous curvature shown in Fig. 5. In the case of the theoretical equation of state (Noya et al., 2007), we report comparisons with the results of calculations using the TIP4P/2005 forcefield, which generally gives densities in closer agreement with experimental values than TIP4P/ice; we find that the theoretical densities are greater (i.e. smaller molecular volume) than our experimental values by $0.50 \%$ at zero pressure, decreasing to $0.25 \%$ at $300 \mathrm{MPa}$.

The c/a ratio exhibits only a very weak dependence on pressure, reflecting the elastic isotropy of the ice Ih structure. We have used the pressure dependence of the elastic constants measured at $237.65 \mathrm{~K}$ by Gagnon et al. (1988) to calculate the change in $c / a$ as a function of pressure in the low-pressure limit $\left(-1.64 \times 10^{-3} \mathrm{GPa}^{-1}\right)$; our experimental results give the pressure dependence of the axial ratio, $(\partial[c / a] / \partial P)_{T}=$ $-1.4(4) \times 10^{-3} \mathrm{GPa}^{-1}$.

\footnotetext{
${ }^{4}$ The relative uncertainty in $K^{S}$ estimated by propagation of the errors on individual elastic constants is $4.8 \%$; in the absence of uncertainties on $\alpha$ and $\gamma$, we have simply propagated this error onto $K^{T}$.
}

5.1.2. Ice II. Ice II has been observed as an accessory phase in several experiments at pressures below $\sim 650 \mathrm{MPa}$. Most notably, it grew in significant quantities after melting of an ADH I-ice IX mixture at $460 \mathrm{MPa}, 179 \mathrm{~K}$ (experiment 1), and data were subsequently collected on cooling from 174 to $139 \mathrm{~K}$, on quenching to $112 \mathrm{~K}$ and upon depressurization. Ice II also forms when ADH II is warmed above $190 \mathrm{~K}$ (experiments 2 and 4) and disproportionates to AMH II + ice II. We have collected data from ice II formed in this way upon cooling from 190 to $155 \mathrm{~K}$ at $550 \mathrm{MPa}$ (experiment 2). If this process runs to completion, then ice II will make up $33.32 \mathrm{~mol} \%$ of the specimen by weight, although we do not know the textural relationship between the ice and ammonia monohydrate: diamond anvil cell observations of ADH disproportionation at much higher pressures indicated that ice formed myrmekitic intergrowths (Johnson et al., 1985). However, a minority phase nucleated in a matrix of another solid crystalline material is likely to be subjected to anisotropic strains.

Fig. 5 shows that our ice II unit-cell volumes collected at different pressures under helium gas are reasonably consistent with the experimental equation of state measured at $225 \mathrm{~K}$ by Fortes et al. (2005) under argon gas. A closer examination of the unit-cell axial ratios provides evidence of considerable strain in the ice II that has formed by exsolution from ADH II. Fig. 7(d) shows the $c / a$ ratios obtained by Fortes et al. (2005) along the $225 \mathrm{~K}$ isotherm, which exhibit a linear dependence on pressure with a slope of $-5.0(2) \times 10^{-3} \mathrm{GPa}^{-1}$. The $c / a$ ratios obtained as a function of temperature at $443 \mathrm{MPa}$ reveal a positive temperature dependence with a slope of $8(2) \times$ $10^{-6} \mathrm{~K}^{-1}$ [compare $1.6(2) \times 10^{-6} \mathrm{~K}^{-1}$ at atmospheric pressure (Fortes et al., 2005)]. When corrected for the difference in temperature, the $c / a$ ratio at $443 \mathrm{MPa}$ agrees very well with the trend observed previously by Fortes et al. (2005). However, the $c / a$ ratio at $550 \mathrm{MPa}$, when similarly corrected (using the $443 \mathrm{MPa}$ temperature coefficient), falls below this trend $(-0.3 \%)$. Moreover, we find that the $c / a$ ratio in the $550 \mathrm{MPa}$ data collected upon cooling from 190 to $155 \mathrm{~K}$ shows no discernible temperature dependence (which we would expect to resolve), also indicative of significant external strain. It is also possible that the anomalous $c / a$ ratio reflects infiltration of helium into the ice II structure, since the work of Lobban et al. (2002) shows that the helium-stuffed ice II structure has a c/a ratio $0.43 \%$ smaller than the helium-free structure at approximately $480 \mathrm{MPa}, 200 \mathrm{~K}$. Finally, ice II observed in the Paris-Edinburgh cell (experiment 6, see Fig. S11) exhibits a $c / a$ ratio (also after correction from 190 to $225 \mathrm{~K}$ using the $443 \mathrm{MPa}$ temperature coefficient) that is consistent with the previously observed trend.

Nonetheless, the one series of temperature-dependent data acquired at $443 \mathrm{MPa}$ provides the first high-pressure measurements of the ice II thermal expansion for comparison with the atmospheric pressure data; previously Lobban et al. (2002) reported an estimate of the high-pressure thermal expansion based on a single measurement at $420 \mathrm{MPa}, 250 \mathrm{~K}$, and a linear interpolation between two other measurements, 280 and $480 \mathrm{MPa}$ at $200 \mathrm{~K}$. 

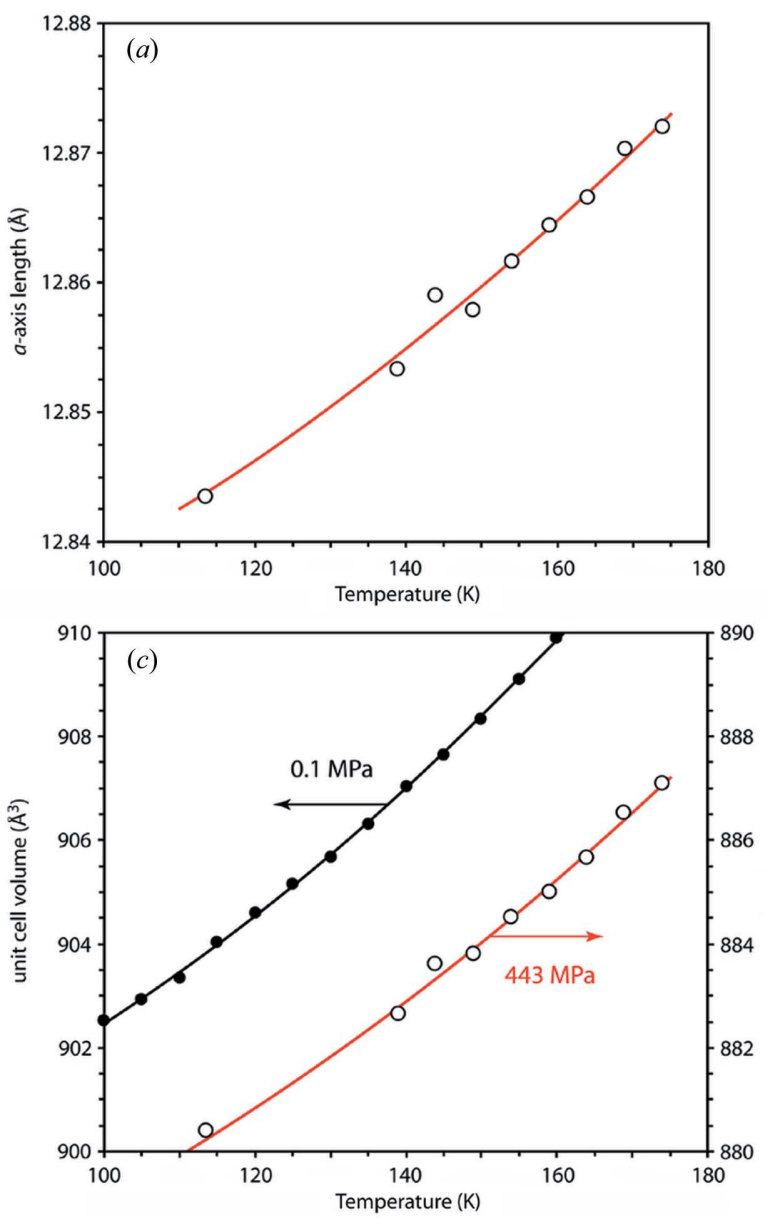
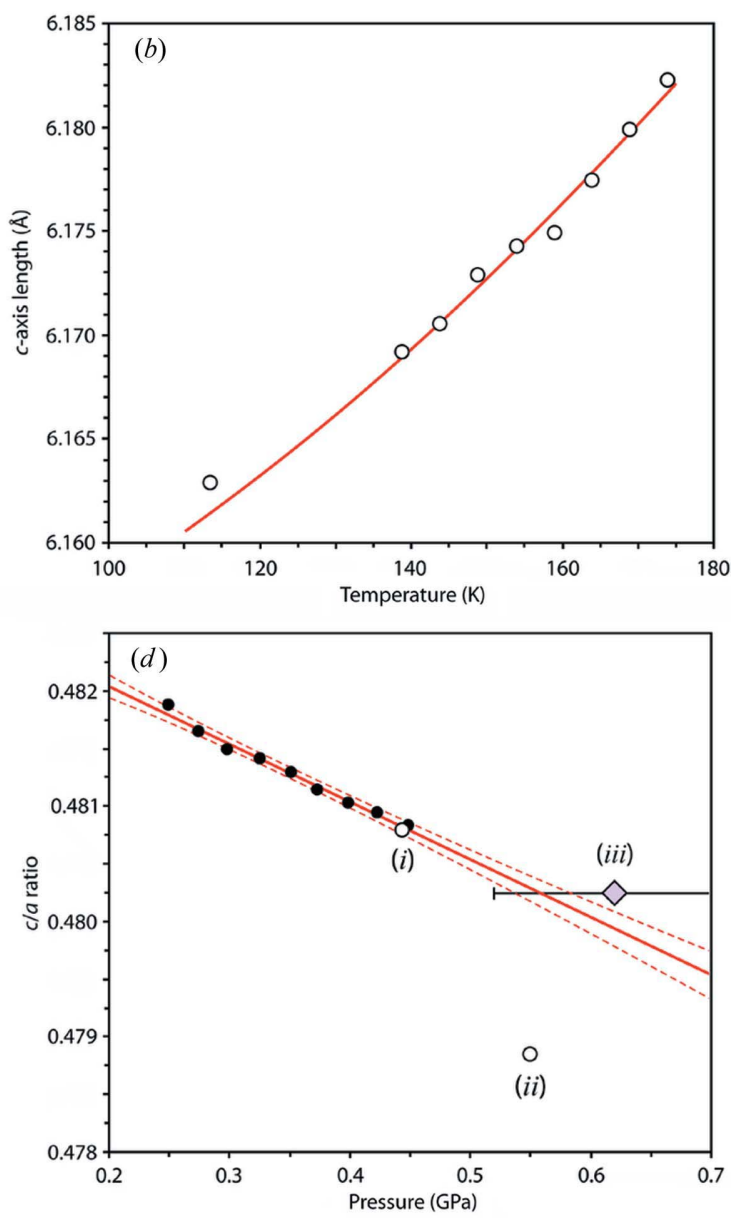

Figure 7

(a) and (b) Refined unit-cell parameters of ice II (triply primitive hexagonal cell) obtained at $443 \mathrm{MPa}$ in experiment 1 . Solid lines are parabolic equations fitted to the data from 139 to $174 \mathrm{~K}$. (c). The unit-cell volumes at $443 \mathrm{MPa}$ (right axis) and at room pressure (left axis, from Fortes et al., 2005). $(d)$ The pressure dependence of the $c / a$ ratio; black circles from Fortes et al. (2005) are fitted with a linear expression (solid line), with $2 \sigma$ confidence limits shown as dashed lines. The temperature-corrected $c / a$ ratios at (i) $443 \mathrm{MPa}$ (experiment 1), (ii) $550 \mathrm{MPa}$ (experiment 2) and (iii) $620 \mathrm{MPa}$ (experiment 6) are shown.

The temperature dependence of the $a$ - and $c$-axis lengths and the unit-cell volume at $443 \mathrm{MPa}$ are shown in Figs. 7(a)7(c), including in Fig. 7(c) the unit-cell volume of ice II at atmospheric pressure. The volume thermal expansion coefficient at $175 \mathrm{~K}, 443 \mathrm{MPa}$ is $\alpha_{V}=155(6) \times 10^{-6} \mathrm{~K}^{-1}$. By comparing this with the extrapolated zero-pressure thermal expansion at $175 \mathrm{~K}, 188(4) \times 10^{-6} \mathrm{~K}^{-1}$, we obtain an average value in the range $0<P<443 \mathrm{MPa}$ of $\partial \alpha_{V} / \partial P=-74(16) \times$ $10^{-6} \mathrm{~K}^{-1} \mathrm{GPa}^{-1}$. Owing to very small systematic offsets between data sets collected at different times (even on the same diffractometer) ${ }^{5}$ we cannot fit a $P-V-T$ surface through these data. However, derivative quantities are not affected by these offsets, and so we may use the data to estimate the pressure dependence of the thermal expansion, $\left(\partial \alpha_{V} / \partial P\right)_{T}$, which in turn is thermodynamically equivalent to the temperature dependence of the isothermal bulk modulus,

\footnotetext{
${ }^{\mathbf{5}}$ These very small systematic offsets are due to millimetre-scale differences in the neutron flight path caused by the difficulty of achieving perfect accuracy in placing the sample in the beam.
}

$\left(\partial K^{T} / \partial T\right)_{P}$, the two properties being related via the isothermal Anderson-Grüneisen parameter, $\delta^{T}$, where

$$
\delta^{T}=\frac{-1}{\alpha_{0} K^{T}}\left(\frac{\partial K^{T}}{\partial T}\right)_{P}=\frac{-K^{T}}{\alpha_{P}}\left(\frac{\partial \alpha_{V}}{\partial P}\right)_{T}
$$

$\alpha_{0}$ and $\alpha_{P}$ are, respectively, the volume thermal expansion at zero pressure and at pressure $=P$. Although equation (6) would appear to require knowledge of the isothermal bulk modulus at the temperature at which $\left(\partial \alpha_{V} / \partial P\right)_{T}$ is evaluated, we note that for a linear dependence of bulk modulus upon temperature, $K^{T}=K_{\text {ref }}^{T}+T(\partial K / \partial T)_{P}$, provided that the bulk modulus is known at some reference temperature, equation (6) can be solved for $\left(\partial K^{T} / \partial T\right)_{P}$. Taking $K_{\text {ref }}^{T}=12.13$ (7) GPa at $225 \mathrm{~K}$ (Fortes et al., 2005), we then solve for the temperature dependence over the full range in which high- and low-pressure data overlap $(110-165 \mathrm{~K})$, finding that $\left(\partial K^{T} / \partial T\right)_{P}=$ $0.13 T-7.6 \mathrm{MPa} \mathrm{K}^{-1}$, with a relative uncertainty of $\sim 25 \%$. Hence, at $165 \mathrm{~K}, \quad\left(\partial K^{T} / \partial T\right)_{P}=-14(3) \mathrm{MPa} \mathrm{K}^{-1}$. For comparison, this value is similar to that in $\mathrm{D}_{2} \mathrm{O}$ ice Ih (Mitz- 
dorf \& Helmreich, 1971), where a linear fit to $K^{S}$ in the range $145-165 \mathrm{~K}$ yields $\left(\partial K^{T} / \partial T\right)_{P}=-9.18$ (4) $\mathrm{MPa} \mathrm{K}^{-1}$.

These values are now used to plot the $P-V-T$ equation of state of ice II (Fig. 6), with the temperature dependence of $V_{0}$ taken from our earlier experimental studies (Fortes et al., 2005), the temperature dependence of $K_{0}$ taken from the foregoing paragraph and $K^{\prime}$ fixed at 6 . MILEOS isotherms are plotted at 175, 190 and $225 \mathrm{~K}$; for comparison, we also draw isotherms at 175, 190 and $225 \mathrm{~K}$ calculated using the TIP4P/ 2005 equation of state (Noya et al., 2007). With the exception of the point at $550 \mathrm{MPa}$ (experiment 2), which exhibits an anomalous $c / a$ ratio, there is good agreement between the TIP4P/2005 EOS and our derived $P-V-T$ equation of state. The ice II molar volume obtained from refinement of data collected in the $\mathrm{P}-\mathrm{E}$ cell corresponds to a pressure of $0.56 \mathrm{GPa}$ (at $190 \mathrm{~K}$ ) using our $P-V-T$ equation of state, which compares favourably to the pressure estimated from the applied load, 0.62 (10) GPa. The theoretical incompressibility (Noya et al., $2007)$ is considerably larger than our experimentally derived value, $K_{0}^{\text {theory }}=15.8 \mathrm{GPa}\left(K^{\prime}=6.75\right)$ versus $K_{0}^{\exp }=12.9 \mathrm{GPa}$ $\left(K^{\prime}=6.00\right)$ at $175 \mathrm{~K}$, and whilst the experimental and theoretical results are in tolerable agreement at lower temperatures, the agreement is much poorer at $225 \mathrm{~K}(\sim 0.7 \%)$.

5.1.3. Ice IX. An ice phase identified initially as either ice III or ice IX (both are tetragonal, space group $P 4_{1} 2_{1} 2$ ) was observed to form when ice Ih co-existing with ADH I was compressed above $\sim 300 \mathrm{MPa}$ (experiments 1 and 4 ), and persisted through the ADH I-II transition at $\sim 475 \mathrm{MPa}$ (see Fig. S3). Unit-cell refinements of this phase were carried out at three points along the $175 \mathrm{~K}$ isotherm using data collected in experiment 1, where the relative abundance was greater than $10 \mathrm{wt} \%$ (in experiment 4 it was much less). We determined that the $c / a$ ratio [1.0013 (1); see Table S2] was characteristic of the proton ordered ice IX phase $(c / a \simeq 1.003)$ rather than the disordered ice III phase $(c / a \simeq 1.040$ ) ( $c f$. Londono et al., 1993). Despite the limited number of data (Figs. 5 and 6) we can test the agreement between experimental and theoretical equations of state.

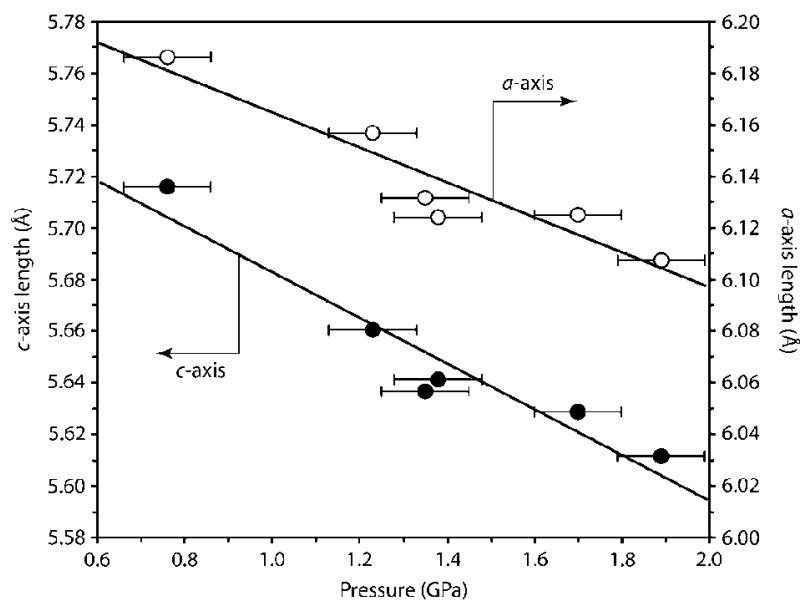

Figure 8

Refined unit-cell parameters of ice VI as a function of pressure $(190<T<$ $200 \mathrm{~K}$ ) from measurements made in the Paris-Edinburgh cell (experiments 6 and 8). Solid lines are weighted linear fits through the data.
Our data are at slightly higher pressures than the range over which Noya et al. (2007) recommend using their TIP4P/2005 theoretical equation of state (220-340 MPa), and higher than $P_{\text {ref }}$ of Choukroun \& Grasset (2007) (355 MPa), which we take to be their upper pressure limit; nonetheless, we have calculated the theoretical density for comparison, represented by the lines marked $(i)$ in Fig. 5. The agreement is very poor indeed in both cases (the calculated densities are off the scale in Fig. 6), being too low by $>5 \%$.

Experimental data on ices III and IX are extremely sparse. We have taken the molar volume at $110 \mathrm{~K}$, atmospheric pressure, $V_{0,110}=307.51(2) \AA^{3}$ (Londono et al., 1993), and applied the volume thermal expansion coefficient estimated at $T=245 \mathrm{~K}, P=250 \mathrm{MPa}$ (Lobban et al., 2000), $\alpha_{V}=239$ (12) $\times$ $10^{-6} \mathrm{~K}^{-1}$, in order to obtain $V_{0,175}$. We have then gone back to the original elastic constants measured in the range 220 $300 \mathrm{MPa}$ at $253 \mathrm{~K}$ (Tulk et al., 1997), re-calculated the bulk moduli ${ }^{6}$ and re-fitted the pressure dependence, finding $K=$ 8 (2) $P+7.6$ (4) GPa. These parameters are sufficient to calculate the molecular volume as a function of pressure (error propagation yields $1 \sigma$ relative uncertainty of $0.21 \%$ at $300 \mathrm{MPa}$, rising to $0.31 \%$ at $480 \mathrm{MPa}$ ). Given that no allowance has been made for any temperature dependence of the bulk modulus, the agreement shown in Fig. 6 is very good (all three points within $1 \sigma$ ), from 0.055 to $0.102 \%$. Indeed, if the subtle difference in slope between our data and the equation of state are to be believed, then our data are consistent with a greater stiffness at lower temperature; a linear fit gives $K=$ $13(1) \mathrm{GPa}$ at $400 \mathrm{MPa}, 175 \mathrm{~K}$, compared with $K=$ 10.8 (8) $\mathrm{GPa}$ at $400 \mathrm{MPa}, 253 \mathrm{~K}$ from the measurements of Tulk et al. (1997). These results are in line with $(\partial K / \partial T)_{P}$ being roughly double the value found above for ice II. It is very clear that there is insufficient empirical data (particularly at low temperatures) on the pressure and temperature dependence of the density of ices III and IX, although there are well known experimental problems associated with metastability relative to ice II which make such measurements quite challenging.

5.1.4. Ice VI. Ice VI (tetragonal, space group $P 4_{2} / n m c$ ) was observed as an accessory phase co-existing with ADH IV at pressures above $700 \mathrm{MPa}$ in most of our experimental runs with the P-E cell (experiments 5-8). Six data sets were suitable for refinement of the ice VI unit cell, covering the pressure range $0.76<P<1.89 \mathrm{GPa}$ at temperatures of $190<$ $T<200 \mathrm{~K}$, with a precision of better than 1 part in $10^{4}$ for the longest integrated data and $\sim 4$ parts in $10^{4}$ for the shortest counts. The molecular volumes obtained from these refinements are shown in Fig. 5 and the pressure dependence of the unit-cell parameters in Fig. 8.

As is the case for many of the ice polymorphs, there is very little extensive measurement of density as a function of pressure and temperature for ice VI. For comparison, we have plotted an equation of state derived by us from experimental

\footnotetext{
${ }^{6}$ From the published elastic stiffnesses, $c_{i j}$, we found the elastic compliances, $s_{i j}$, by matrix inversion and calculated the axial moduli, $K_{a}=\left(s_{11}+s_{12}+s_{13}\right)^{-1}$ and $K_{c}=\left(2 s_{13}+s_{33}\right)^{-1}$, to obtain the relaxed bulk modulus, $K^{-1}=2 K_{a}^{-1}+$ $K_{c}^{-1}$.
} 
quantities, and one obtained from theory. We have not drawn any comparison with the equation of state for ice VI of Choukroun \& Grasset (2007) since they present a model with no pressure dependence of the density (both pressure coefficients, $a_{P 2}$ and $a_{P 3}$, are equal to 0 ). The theoretical equation of state is based on the TIP4P/2005 forcefield (Noya et al., 2007), and the agreement is good (between $0.06-0.69 \%$ ). As we did for ice IX above, we have used the measured elastic constants of ice VI in the range 620-820 MPa at $271 \mathrm{~K}$ (Tulk et al., 1997) to determine the bulk modulus and its pressure dependence, finding $K=6.7$ (9) $P+13.1$ (6) GPa. A reference volume was found at $1.1 \mathrm{GPa}, 175 \mathrm{~K}$ by fitting a parabolic expression through the measurements of Kuhs et al. (1984) along the $1.1 \mathrm{GPa}$ isobar: $V_{1.1,175}=217.12(7) \AA^{3}$. The agreement is much poorer than with the theoretical equation of state; it is clearer in this instance than it was for ice IX that the cause of the disagreement is stiffening of the bulk modulus at lower temperatures. We have fitted a weighted MILEOS [equation (5)] through our data [with $K_{0,175}^{\prime} \equiv 6.7$ from the Brillouin scattering measurements of Tulk et al. (1997)], to obtain $V_{0,175}=227(1) \AA^{3}$ and $K_{0,175}=18$ (2) GPa. This zero-pressure volume compares well with that of ice VI quenched to atmospheric pressure at $98 \mathrm{~K}, V_{0,98}=227.6(8) \AA^{3}$ (Kamb, 1965). The bulk modulus, and its first pressure derivative, from the theoretical equation of state (Noya et al., 2007) are $K_{0,175}=$ $19.30 \mathrm{GPa}$ and $K_{0,175}^{\prime}=6.60$. Once again, the apparent change in bulk modulus is consistent with a value of $(\partial K / \partial T)_{P}$ a few times larger than that obtained for ice II above (order of a few tens of $\mathrm{MPa} \mathrm{K}^{-1}$ ).

Fig. 8 shows the pressure dependence of the unit-cell edge lengths. Weighted linear fits through these data allow us to obtain very crude estimates of the axial incompressibilities. At $1 \mathrm{GPa}, K_{a}=-a(\partial P / \partial a) \simeq 91(8) \mathrm{GPa}$ and $K_{c} \simeq 66(6) \mathrm{GPa}$. Since our fitted MILEOS gives a bulk modulus of 25 (2) GPa at $1 \mathrm{GPa}$, these axial incompressibilities roughly satisfy the requirement (for a tetragonal crystal) that $K^{-1}=$ $2 K_{a}^{-1}+K_{c}^{-1}=27$ (2) GPa. Not only are our axial bulk moduli larger than those calculated from the $271 \mathrm{~K}$ elastic constants of Tulk et al. (1997), as one would expect owing to the temperature difference, but the difference between them both absolute and relative - is much greater. Our results suggest that the temperature dependence of the $a$-axis elastic modulus is roughly twice as large as that of the $c$ axis.

\subsection{ADH polymorphs}

The densities reported below are summarized in Fig. 9.

5.2.1. ADH I. Our analysis of the compression of ADH I in experiment 1 was presented by Fortes, Wood, Knight et al. (2003). However, in order to provide a complete picture of the behaviour of ammonia dihydrate we show this data in Fig. 9 and present the coefficients of a MILEOS [equation (5)] fit to nine points measured in the range $41.1<P<450.4 \mathrm{MPa}$ along the $175 \mathrm{~K}$ isobar; $V_{0,175}=365.3$ (2) $\AA^{3}, K_{0,175}=7.5$ (3) GPa and $K_{0,175}^{\prime}=7$ (1). This empirical equation of state permits direct comparison of the elastic properties of various ADH poly- morphs and determination of the volume difference between phases I and II.

5.2.2. ADH Ila. ADH II has been observed over only a relatively narrow pressure interval (440-550 MPa), and any one experiment has only collected data over part of this range, as shown in Fig. 9. Although we have fitted equation (5) to the unit-cell volumes obtained in this pressure range, the parameters are very poorly determined. Fixing $K_{0,175}^{\prime} \equiv 7$ yields $V_{0,175}=334$ (4) $\AA^{3}$ and $K_{0,175}=5$ (1) GPa. This bulk modulus is softer than that of the lower-pressure polymorph, ADH I, which is certainly possible (ice III has a smaller bulk modulus than ice $\mathrm{Ih}$ ), but uncommon. Reducing $K^{\prime}$ forces $K_{0}$ to become stiffer, but it is still smaller than that of ADH I when $K^{\prime}>1.5$. It is more likely that the available data are too limited to constrain the incompressibility. Nevertheless, the sparse data provide an adequate estimate of the density of ADH in this pressure range, and of the volume difference between ADH I and II $(\Delta V / V=-10.7 \%)$.

Sufficiently long integrations were made for refinement of ADH II $a$ unit-cell parameters as a function of temperature in experiment 1 (Fig. 10), cooling slowly from 174 to $139 \mathrm{~K}$ (eight data points at $5 \mathrm{~K}$ intervals) along the $443 \mathrm{MPa}$ isobar, followed by a rapid quench to $112 \mathrm{~K}$ and subsequent reduction of pressure. Le Bail structureless profile refinements were used to determine the temperature dependence of the unitcell parameters, $a, b, c, \beta$ and $V$. Inspection of the data showed that the axial ratios (referred to three orthogonal axes, $a \sin \beta$, $b$ and $c$ ) changed linearly during the slow cooling period, but depart substantially from this trend after quenching. This is also manifested in the b/a ratio of AMH II (see below). As such, when we have fitted the unit-cell parameters, we have used only the slow-cool data (although the quench data points are shown). This distortion of a higher-temperature unit-cell

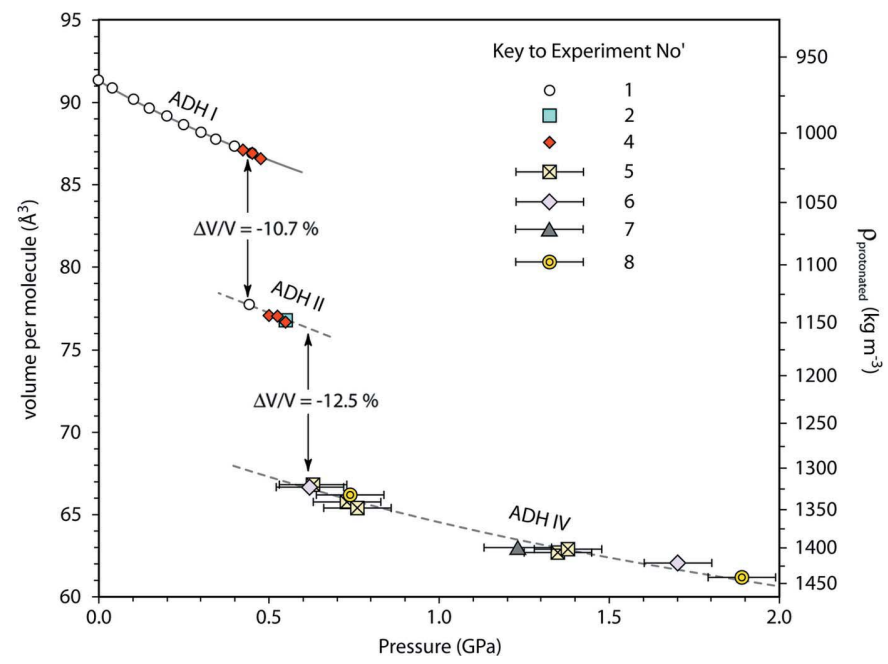

Figure 9

Molecular volumes (left axis) and densities (right axis) of the ADH polymorphs observed during our studies. The solid line through the ADH I data (experiment 1) and the dashed line through the ADH IV data (experiments 5-8) are Murnaghan integrated linear equations of state [equation (5), see text]. The dashed line through the ADH II data is simply a guide to the eye, the slope being fixed equal to that of ADH I at $500 \mathrm{MPa}$. 
shape after quenching is suggestive of a frozen-in dynamical relaxation process (such as orientational ordering of hydrogen bonds) and we have observed a similar phenomenon in rapidly quenched mirabilite $\left(\mathrm{Na}_{2} \mathrm{SO}_{4} \cdot 10 \mathrm{D}_{2} \mathrm{O}\right.$; Brand et al., 2009). The unit-cell parameters $a, b, c, \beta$ and $V$ have all been fitted with a simple parabolic equation of the form $a+b T^{2}$ (Fig. 10), which satisfies the requirement for the thermal expansion to tend to zero in the low-temperature limit; for the purpose of simple density calculations, the molar volume is well represented in the temperature range $110<T<175 \mathrm{~K}$ by this expression with coefficients $a=45.71(2) \mathrm{cm}^{3} \mathrm{~mol}^{-1}$ and $b=3.57(7) \times$ $10^{-7} \mathrm{~cm}^{3} \mathrm{~mol}^{-1} T^{-2}$.

The volume coefficient of thermal expansion for ADH II $a$ at $443 \mathrm{MPa}, 175 \mathrm{~K}$ is $\alpha_{V} \simeq 283(7) \times 10^{-6} \mathrm{~K}^{-1}$, which is identical (within errors) to the $0.1 \mathrm{MPa}$ value for $\mathrm{ADH}$ I at the same temperature, $\alpha_{V} \simeq 281 \times 10^{-6} \mathrm{~K}^{-1}$ (Fortes, Wood, Knight et al., 2003), and significantly larger than those for ice Ih $\left(\alpha_{V}=108 \times 10^{-6} \mathrm{~K}^{-1}\right.$ for $\mathrm{D}_{2} \mathrm{O}$; Röttger et al., 1994), ice II
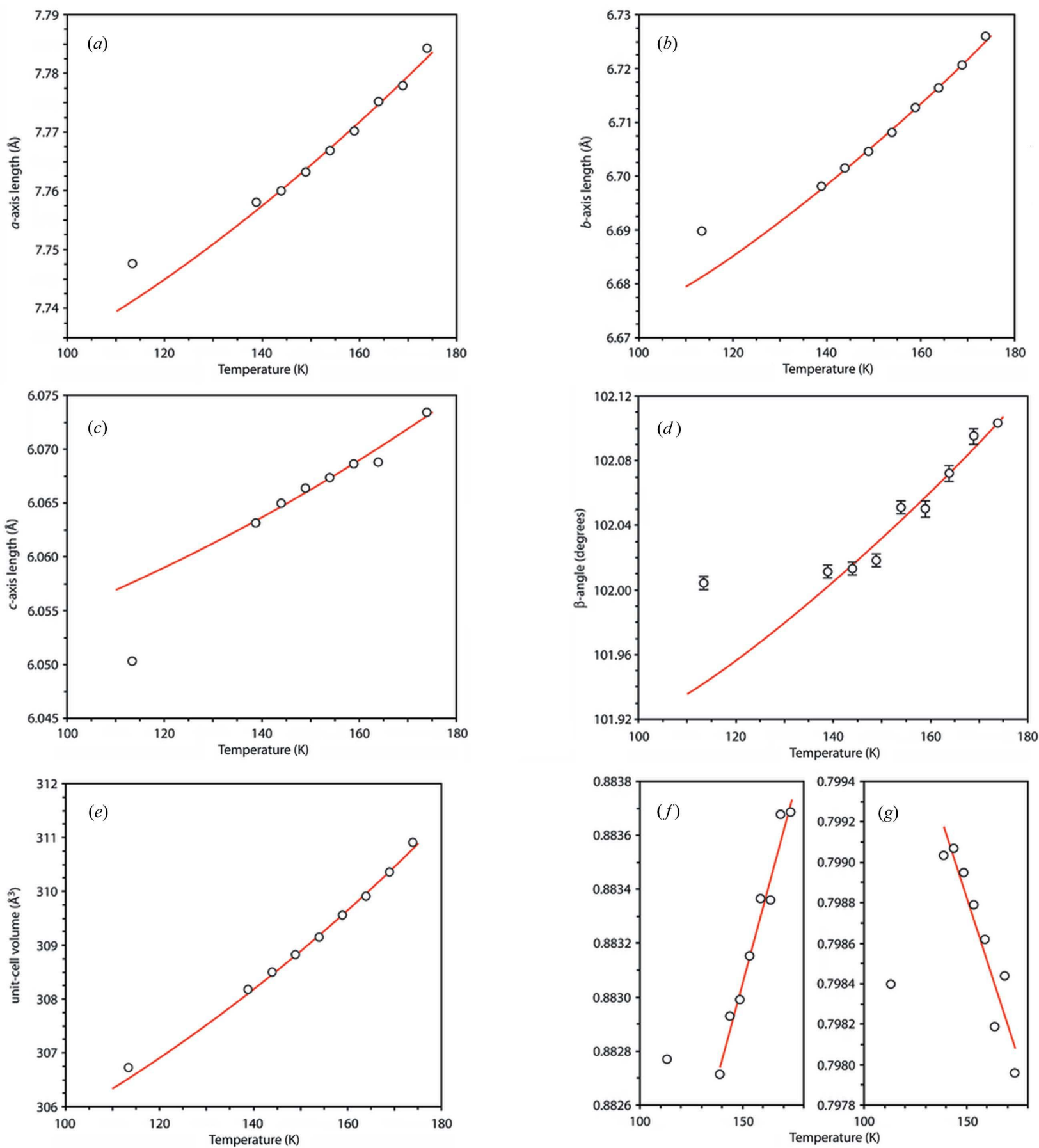

Figure 10

$(a)-(d)$ The refined unit-cell parameters of ADH II $a$ obtained at $443 \mathrm{MPa}$ in experiment 1. (e) The unit-cell volume. Solid lines are parabolic equations fitted to the data from 139 to $174 \mathrm{~K}$. The temperature dependencies of the orthogonalized axial ratios are shown in $(f)$ and $(g)$. 
$\left(155 \times 10^{-6} \mathrm{~K}^{-1}\right.$, see above $)$ or AMH II $\left(177 \times 10^{-6} \mathrm{~K}^{-1}\right.$, see below), all at the same temperature.

The axial ratio $b /(a \sin \beta)$ decreases at a rate of $3.0(2) \times$ $10^{-5} \mathrm{~K}^{-1}$ on cooling from 174 to $139 \mathrm{~K}$, and the ratio $c /(a \sin \beta)$ increases at a rate of $3.0(4) \times 10^{-5} \mathrm{~K}^{-1}$. These changes in axial ratios reflect anisotropy of the thermal expansion; the expansivities of the three orthogonal axes are estimated from the simple polynomial fits, from which we obtain $\alpha_{a \sin \beta} \simeq$ $96(4) \times 10^{-6} \mathrm{~K}^{-1}, \alpha_{b} \simeq 132(3) \times 10^{-6} \mathrm{~K}^{-1}$ and $\alpha_{c} \simeq 52(4) \times$ $10^{-6} \mathrm{~K}^{-1}$ [note that $\alpha_{V}=\alpha_{a \sin \beta}+\alpha_{b}+\alpha_{c}=280(6) \times$ $10^{-6} \mathrm{~K}^{-1}$ ]. The thermal expansion along the twofold axis is thus comparatively large, and that along the $c$ axis quite small, and this will reflect the intermolecular connectivity of the crystal structure. We would expect the twofold axis to exhibit the highest axial compressibility and the $c$ axis the lowest axial compressibility.

Data were collected from $\mathrm{ADH}$ II $a$, and a mixture of II $a$ and II $b$, in experiments 4 and 2, respectively. However, these span only a narrow temperature range $(<15 \mathrm{~K})$, and the integration times were very short, resulting in very large uncertainties on the unit-cell parameters. Further measurements of the pressure and temperature dependencies of the $\mathrm{ADH} \mathrm{II} a$ and II $b$ unit-cell parameters are required, as well as
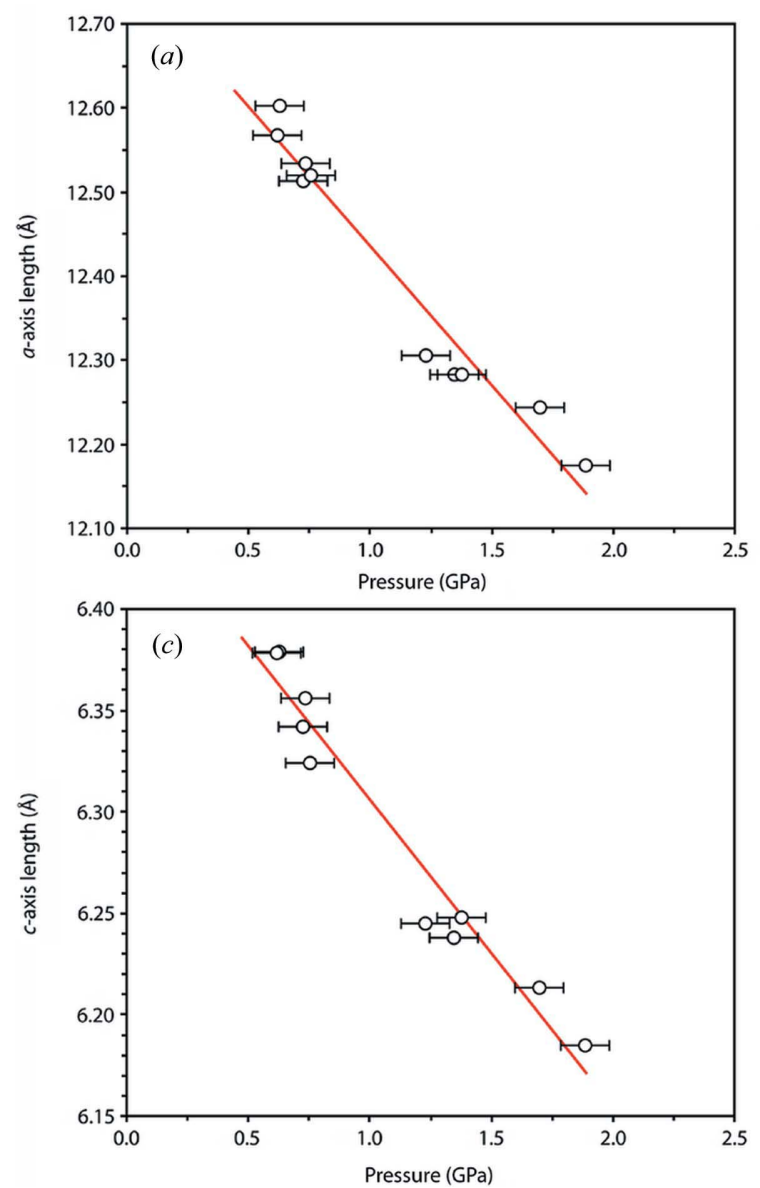

an investigation of the possible structural differences between the two phases and a comparison of possible proton siteoccupancy ordering during slow cooling and rapid quenching.

5.2.3. ADH IV. ADH IV has been observed only in the P-E cell (experiments 5-8), and diffraction data have been integrated at a range of pressures and temperatures. In no instance do we possess diffraction patterns of phase-pure ADH IV; each of our specimens contain either accessory ice II, ice VI, $\mathrm{AMH}$ II or the $\mathrm{Pb}$ pressure calibrant. With increasing load, the Bragg reflections become progressively more strain broadened by nonhydrostatic stresses. In consequence, data suitable for extracting the unit-cell parameters by Le Bail profile refinement are restricted to pressures below $\sim 2 \mathrm{GPa}$. This data set contains ten diffraction patterns collected in the ranges $0.62<P<1.89 \mathrm{GPa}$ and $190<T<200 \mathrm{~K}$, with integration times ranging from $30 \mathrm{~min}$ to $13 \mathrm{~h}$ (experiments 5-8). These allowed us to refine the unit-cell parameters of ADH IV with a precision of between 5-8 parts in $10^{5}$ for the longer counts (e.g. Table S3) and 3-4 parts in $10^{4}$ for the shortest counts.

The molecular volume (and density) of $\mathrm{ADH}$ IV as a function of pressure are shown in Fig. 9; the uncertainty in pressure is of order $\pm 0.1 \mathrm{GPa}$, and the errors on the volume
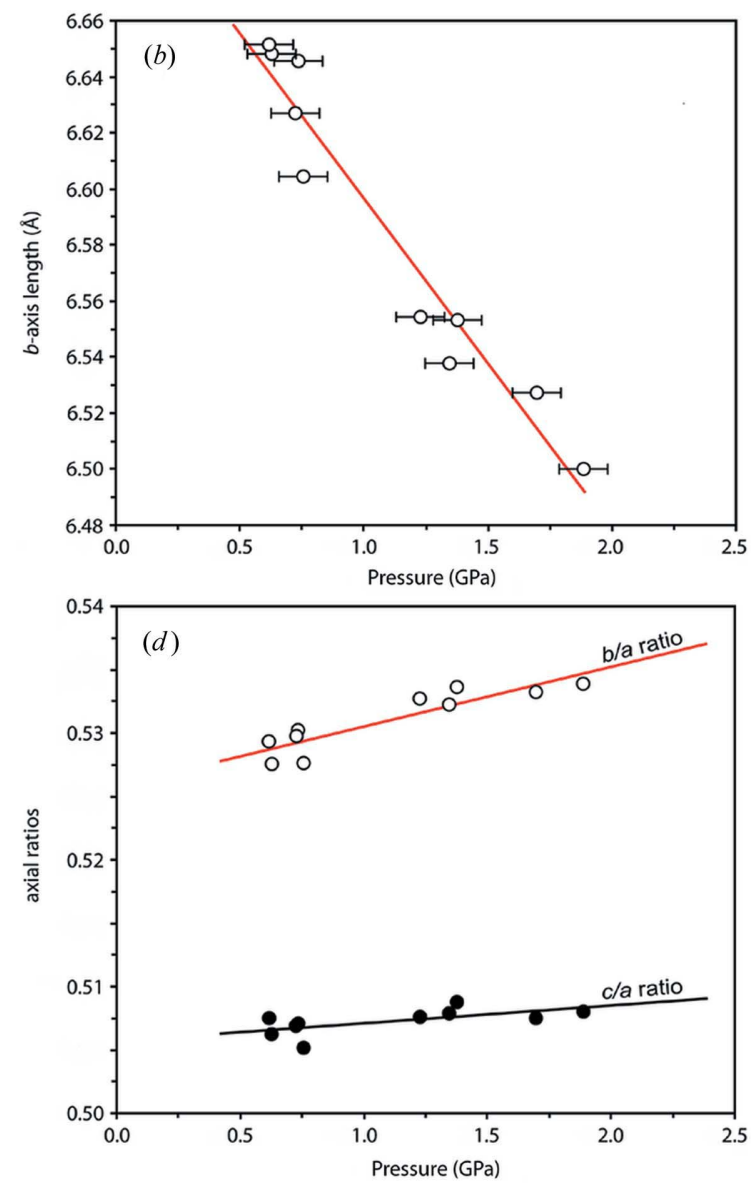

Figure 11

$(a)-(c)$ The refined unit-cell parameters of ADH IV as a function of pressure (experiments 5-8). Solid lines are weighted linear fits to the data. The pressure dependencies of the axial ratios are shown in $(d)$. 
are smaller than the symbols. A free refinement of all parameters in equation (5) to these data yields very large standard errors. Fixing $K^{\prime} \equiv 6$ yields $V_{0}=568(2) \AA^{3}$ and $K_{0}=$ 8.0 (5) GPa; this fit is illustrated by the dashed line through the ADH IV data in Fig. 9. The zero-pressure bulk modulus is the same (within errors) as that found for $\mathrm{ADH}$ I (see above), although reducing $K^{\prime}$ stiffens $K_{0}$ [for $K^{\prime} \equiv 5, K_{0}=9.2$ (5) GPa]. Although the density of ADH IV is comparable to that of ice VI (compare Fig. 5), and it is stable over a similar pressure range, it is considerably softer ( $c f$. Gagnon et al., 1990; Tulk et al., 1997).

Fig. 11 shows the pressure dependence of the unit-cell edge lengths and of the axial ratios $b / a$ and $c / a$. Weighted linear fits through these data allow us to obtain very crude estimates of the axial incompressibilities. At $1 \mathrm{GPa}, K_{a}=-a(\partial P / \partial a) \simeq$ 40 (2) GPa, $K_{b} \simeq 56$ (3) GPa and $K_{c} \simeq 42$ (2) GPa. Since our fitted MILEOS gives a bulk modulus of 14.0 (5) GPa at $1 \mathrm{GPa}$, these axial incompressibilities roughly satisfy the requirement (for an orthorhombic crystal) that $K^{-1}=K_{a}^{-1}+K_{b}^{-1}+K_{c}^{-1}=$ 15.2 (4) GPa. The very weak pressure dependence of the $c / a$ ratio is due to the close similarity in the incompressibility of the $c$ and $a$ axes.

Note that fitting to data obtained from a series of separate loadings is not formally correct, since each loading has some unknown systematic error associated with small shifts in sample position. Moreover, these data are not strictly isothermal although the influence of temperature over only a $10 \mathrm{~K}$ range will not be large.

\subsection{AMH polymorphs}

The densities reported below are summarized in Fig. 12. Although we have collected no data on AMH I, we show as a solid line the equation of state reported by Loveday \& Nelmes (2004) fitted to data measured in the range $0<P<3 \mathrm{GPa}, 130$ $<T<150 \mathrm{~K}$, which has MILEOS parameters $V_{0}=247.66 \AA^{3}$, with $K_{0} \equiv 8.9$ (4) GPa, $K^{\prime}=4.2$ (3).

5.3.1. AMH II. AMH II, like ADH II, has been observed over only a relatively narrow pressure interval (440-550 MPa), and no one experiment has collected data as a function of pressure, only of temperature. However, our results prove unequivocally that the unit cell of AMH II must contain 16 formula units. Indeed, as Fig. 12 shows, even if $Z=15$ (which is most unlikely), the density will be less than AMH I, and a more plausible next-smallest value of $Z=12$ is thus ruled out entirely. However, for $Z=16$, a reasonable density increase is realized, with the volume change at $400 \mathrm{MPa}$ being approximately $-6 \%$. There are insufficient data to fit equation (5) usefully, and so the dashed line through our measurements in Fig. 12 is a grossly approximated MILEOS with $K_{0,175} \equiv$ $11 \mathrm{GPa}, K_{0,175}^{\prime} \equiv 5$ and the zero-pressure volume varied to obtain the best fit by eye, $V_{0,175}=930.4 \AA^{3}$.

Sufficiently long integrations were made for refinement of AMH II unit-cell parameters as a function of temperature in experiments 1 and 2 (Fig. 13). In the former, the sample was cooled slowly from 174 to $139 \mathrm{~K}$ (eight data points at $5 \mathrm{~K}$ intervals) along the $443 \mathrm{MPa}$ isobar, followed by a rapid quench to $112 \mathrm{~K}$ and subsequent reduction of pressure, and in the latter the sample was cooled slowly from 190 to $155 \mathrm{~K}$ (also eight points at $5 \mathrm{~K}$ intervals) along the $550 \mathrm{MPa}$ isobar. Le Bail refinements were used to determine the temperature dependence of the unit-cell parameters, $a, b, c$ and $V$. Inspection of the data showed that the $b / a$ axial ratio changed linearly during the slow cooling period ( $c / a$ exhibits only a very weak temperature dependence) but departed substantially from this trend after quenching. As for ADH II, we have fitted the unit-cell parameters using only the slow-cool data.

The unit-cell parameters $a, b, c$ and $V$ at both pressures have been fitted with a simple parabolic equation of the form $a+$ $b T^{2}$ (Fig. 13). The volume coefficient of thermal expansion for AMH II at $443 \mathrm{MPa}, 175 \mathrm{~K}$ is $\alpha_{V} \simeq 177(6) \times 10^{-6} \mathrm{~K}^{-1}$, and at $550 \mathrm{MPa}, 175 \mathrm{~K}$ is $\alpha_{V} \simeq 162(4) \times 10^{-6} \mathrm{~K}^{-1}$, yielding $\partial \alpha_{V} / \partial P=$ $-139(65) \times 10^{-6} \mathrm{~K}^{-1} \mathrm{GPa}^{-1}$. Whilst the absolute value of the thermal expansion coefficient is similar to that observed in ice II, the pressure dependence is roughly twice as large. Following the method employed in $\$ 5.1 .2$ for ice II, we can use $\partial \alpha_{V} / \partial P$ to infer the magnitude of $\partial K / \partial T$ [equation (6)]. Since we do not know the bulk modulus, we derive an expression for the variation of $\partial K / \partial T\left(\mathrm{MPa} \mathrm{K}^{-1}\right)=3.65 K-21.5$, with $K$ in units of $\mathrm{GPa}$, the relative uncertainty being $\sim 50 \%$. Hence, for $K=12 \mathrm{GPa}, \partial K / \partial T=-22(11) \mathrm{MPa} \mathrm{K}^{-1}$, which is once again very similar to the values obtained for ice II.

The expansivities of the three orthogonal axes are estimated from the simple parabolic fits, from which we obtain (at $443 \mathrm{MPa}, 175 \mathrm{~K}) \alpha_{a} \simeq 51(3) \times 10^{-6} \mathrm{~K}^{-1}, \alpha_{b} \simeq 70(1) \times$ $10^{-6} \mathrm{~K}^{-1}$ and $\alpha_{c} \simeq 56(3) \times 10^{-6} \mathrm{~K}^{-1}$ [note that $\alpha_{V}=\alpha_{a}+\alpha_{b}+$ $\alpha_{c}=177$ (4) $\left.\times 10^{-6} \mathrm{~K}^{-1}\right]$. The variation in $b / a$ with temperature is $\sim 20$ times larger than the variation in $c / a$, in agreement with the close similarity in $\alpha_{a}$ and $\alpha_{c}$.

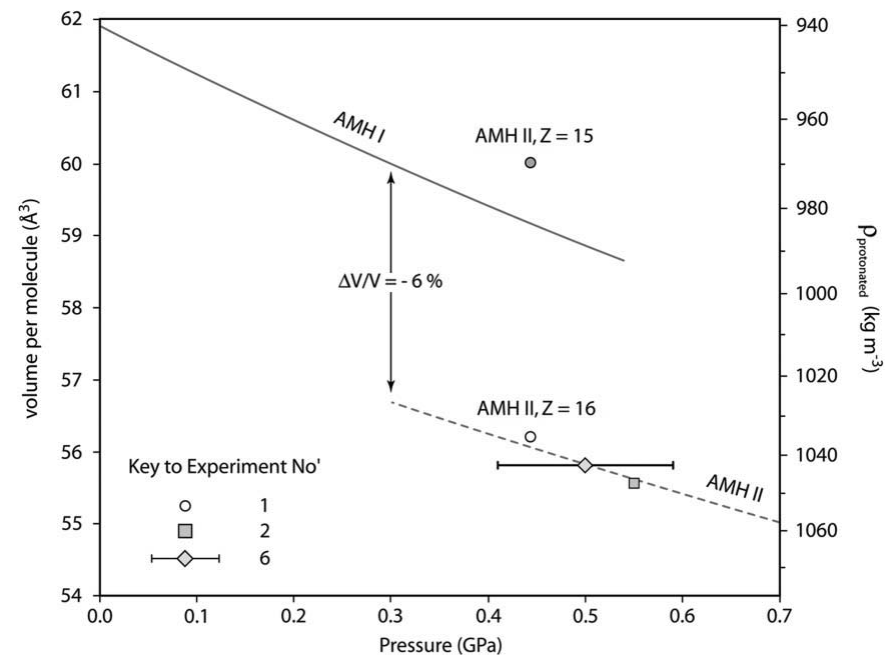

Figure 12

Molecular volumes (left axis) and densities (right axis) of AMH I [solid line is a MILEOS plotted from parameters given by Loveday \& Nelmes (2004)] and AMH II (this work). The open circle shows the density of AMH II assuming 16 formula units per unit cell, and the filled circle is for $Z=15$ at the same pressure. The curve for AMH I corresponds to temperatures of $130-150 \mathrm{~K}$, whereas the curve for AMH II corresponds to $175 \mathrm{~K}$. 


\section{Results IV: the high-pressure phase diagram}

\subsection{The $P-T$ phase diagram of ammonia dihydrate $\left(X_{\mathrm{NH}_{3}}=\right.$ $33.3 \mathrm{~mol} \% \mathrm{NH}_{3}$ )}

Generally speaking, in crystallographic experiments one strives to avoid melting of the specimen, since this may either result in it leaking from the sample holder or else ruin a crystalline powder which took considerable effort to prepare.
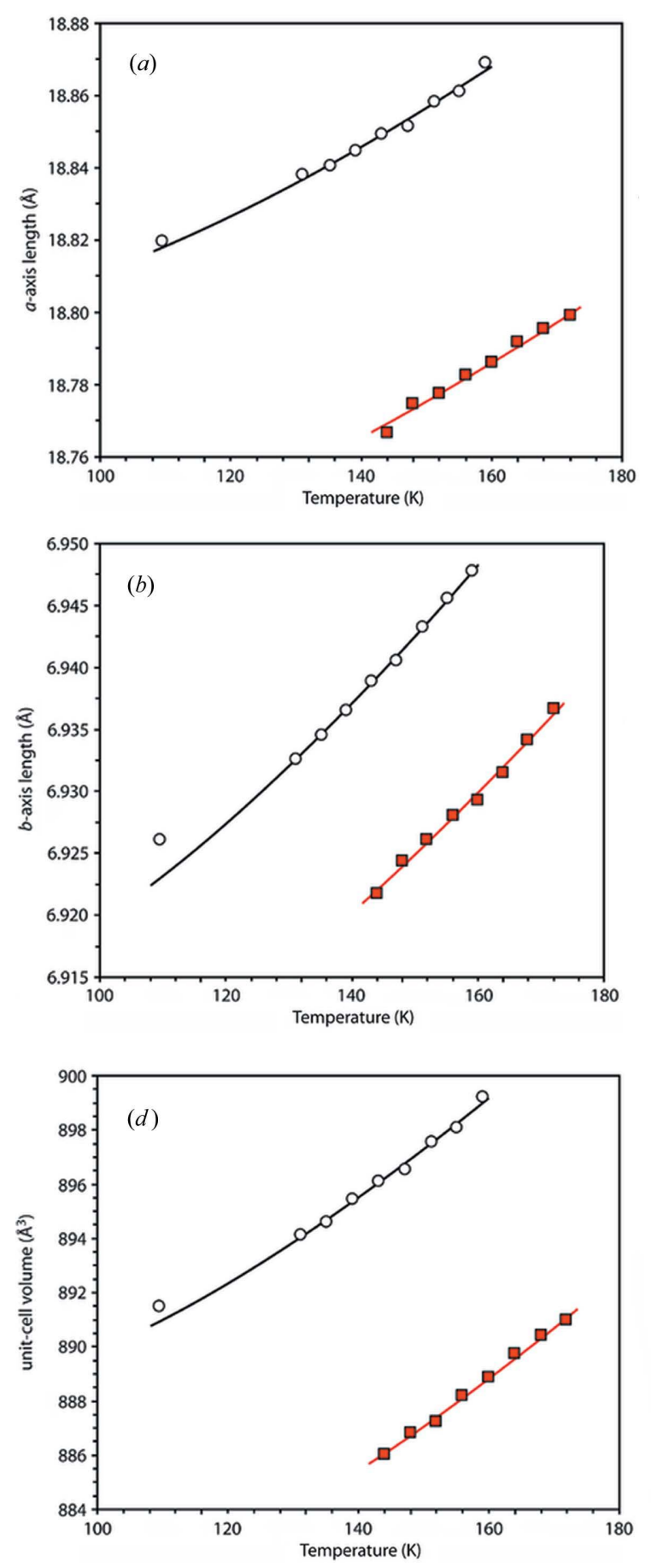

However, we have melted specimens of ADH + ice and AMH + ice at a range of pressures (Fig. 14): in experiment 1 we very likely observed a metastable extension of the ADH I melting line between 174 and $179 \mathrm{~K}$ at $460 \mathrm{MPa}$; in experiment 4 we observed partial melting of AMH II + ice II to ice II + liquid at $196 \mathrm{~K}, 550 \mathrm{MPa}$; and in experiment 7 we observed partial melting of ADH IV + ice VI to ice VI + liquid between 235 and $240 \mathrm{~K}$ at $1.23 \mathrm{GPa}$. These values are for deuterated samples, and the equivalent points in the protonated system may be $2-5 \mathrm{~K}$ lower in temperature at these pressures.

The melting curve of ADH I is known to be quite flat as a function of pressure. A polynomial fit through the data of Hogenboom et al. (1997) in the range $0<P<315 \mathrm{MPa}$ yields an extrapolated $T_{\mathrm{m}} \simeq 172$ (1) $\mathrm{K}$ at $460 \mathrm{MPa}$, depending on the order of polynomial fitted (compare $176.09 \mathrm{~K}$ at atmospheric pressure). Our specimen in experiment 1 was very likely within a degree of its melting point when we began to raise the temperature at this pressure.

Hogenboom et al. (1997) also published a melting curve up to $\sim 1.2 \mathrm{GPa}$, yielding $T_{\mathrm{m}} \simeq 197 \mathrm{~K}$ at $550 \mathrm{MPa}$ and (extrapolated slightly) $T_{\mathrm{m}} \simeq 231 \mathrm{~K}$ at $1.23 \mathrm{GPa}$. Their curve was fitted on the assumption that a single liquidus phase exists
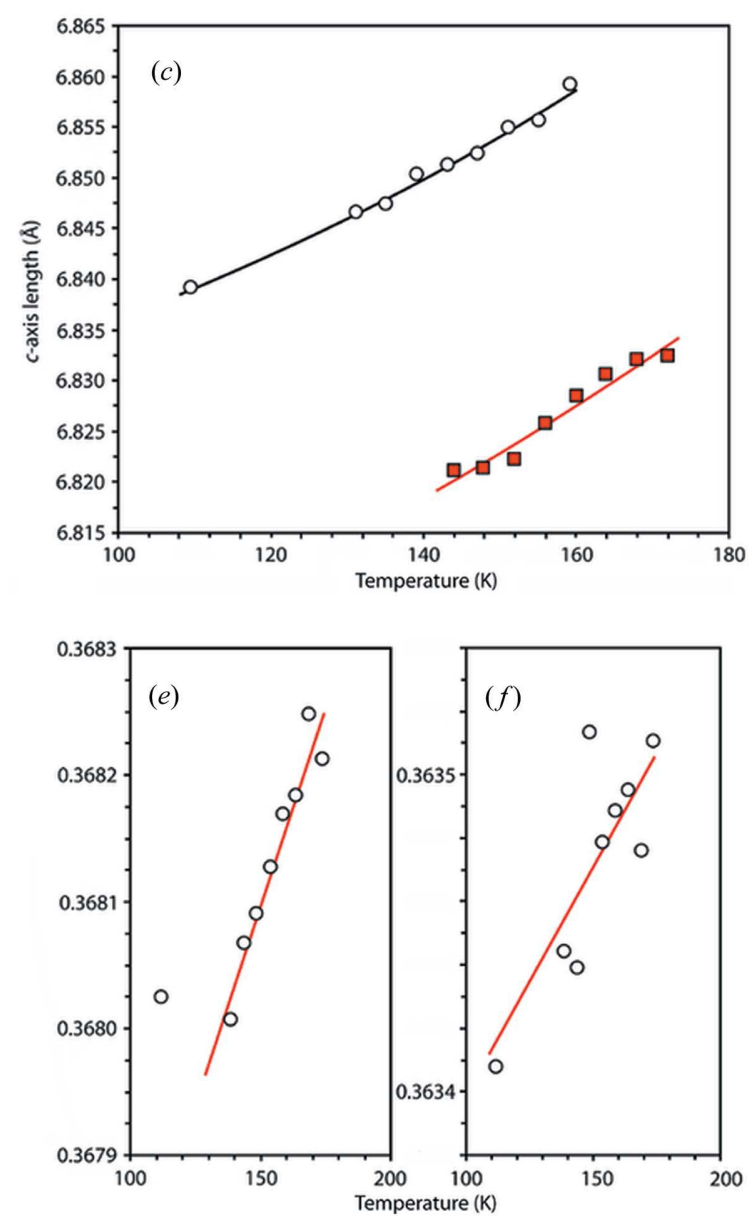

Figure 13

(a)-(c) The refined unit-cell parameters of AMH II obtained at $443 \mathrm{MPa}$ (open circles) and $550 \mathrm{MPa}$ (filled squares) in experiments 1 and 2, and (d) the unit-cell volumes. Solid lines are parabolic equations fitted to the data from 139 to $174 \mathrm{~K}$ (443 MPa) and 155 to $190 \mathrm{~K}$ (550 MPa). The temperature dependencies of the axial ratios at $443 \mathrm{MPa}$ are shown in $(e)$ and $(f)$. 
over this pressure range, which we have shown to be incorrect. A melting curve inflected at $\sim 600 \mathrm{MPa}$ (above which ADH IV is stable) such that $T_{\mathrm{m}} \simeq 193 \mathrm{~K}$ (protonated) at $550 \mathrm{MPa}$ agrees quantitatively with our observation (Fig. 14). Given the uncertainties in pressure calibration $( \pm 0.1 \mathrm{GPa})$, our observation at $1.23 \mathrm{GPa}$ agrees well with the measurements of Hogenboom et al. (1997).

\subsection{The $T-X$ phase diagram at $P=550 \mathrm{MPa}$}

Fig. 15 shows our interpretation of the phase transitions observed to occur upon warming of an $\mathrm{ADH}+$ ice mixture at $550 \mathrm{MPa}$. The breakdown ADH II $\rightarrow$ ice II + AMH II occurs at $188-190 \mathrm{~K}$ (experiments 2 and 4), followed by partial melting to ice II + liquid at $196 \mathrm{~K}$. The breakdown of ADH II is probably a kinetically controlled process, limited by molecular diffusion; hence our observation merely indicates the temperature at which this process runs to completion on the timescale of our diffraction pattern integrations. Partial melting occurs at a simple binary eutectic in the system waterammonia monohydrate. We have inferred the shape of the ice liquidus by fitting a quadratic expression to three points, these being the melting point of pure $\mathrm{D}_{2} \mathrm{O}$ ice $\mathrm{V}$ at $550 \mathrm{MPa}$ (271.06 K; Bridgman, 1935); our observation of the ice II liquidus at $\sim 210 \mathrm{~K}, 30 \mathrm{wt} \%$ ammonia (determined from the abundances of ADH and ice in the specimen); and a eutectic at $196 \mathrm{~K}$, the composition of which was varied to give the closest agreement with the observed liquidus curvature in the data of Hogenboom et al. (1997) at $300 \mathrm{MPa}$. The resulting ice II (ice V above $217 \mathrm{~K}$ ) liquidus is defined as $T_{\mathrm{m}}=-0.0344 X^{2}-$ $1.0021 X+271.06$, where $X$ is the wt $\%$ ammonia concentration. Once again, the liquidus in the protonated system will be

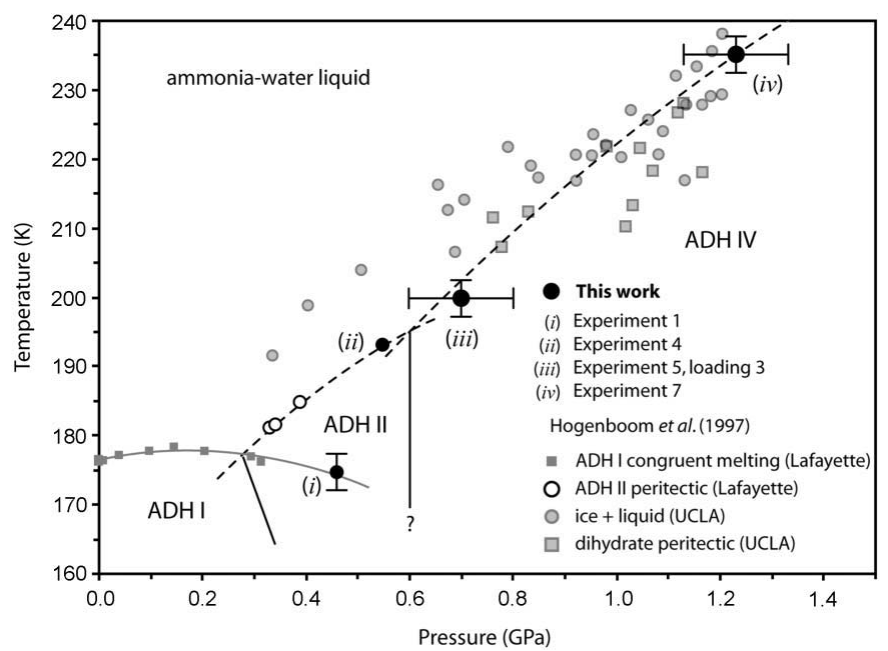

Figure 14

Pressure dependence of the ADH melting line, showing data published by Hogenboom et al. (1997) and the various observations of melting reported in this work (all reduced by $3 \mathrm{~K}$, the likely difference between protonated and deuterated systems). The grey ADH I congruent melting line is a quadratic fit through the data of Hogenboom et al. (1997) and our metastable melting point at $460 \mathrm{MPa}$ (experiment 1). The dashed ADH II and IV melting lines are rough guides to the eye. Our ADH IV data include the confirmed partial melting at $1.23 \mathrm{GPa}$ (experiment 7) as well as the observation of pressure freezing at $\sim 700 \mathrm{MPa}$ (experiment 5 , loading 3).
$1-2 \%$ lower in temperature. The eutectic composition is quite narrowly constrained by the plausible range of liquidus curvatures to be within $\pm 1 \mathrm{wt} \%$ of $34.4 \mathrm{wt} \%$ ammonia.

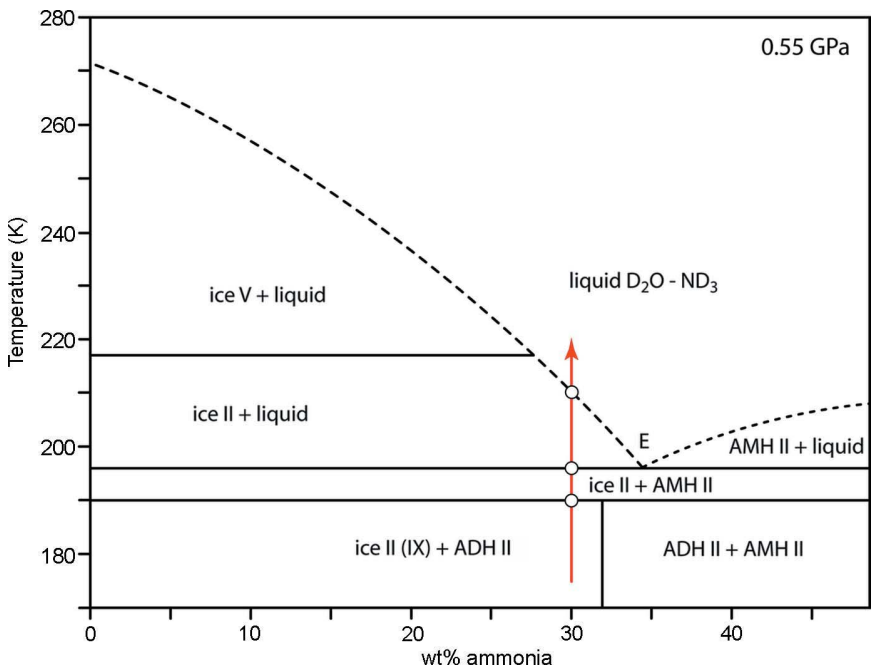

Figure 15

(Experiments 2 and 4.) Derived $T-X$ slice through the $P-T-X$ phase diagram at $550 \mathrm{MPa}$, showing phase boundaries consistent with the sequence of transitions observed on warming at this pressure (red line), beginning with disproportionation of ADH (+ accessory ice) to ice II + AMH II at around $190 \mathrm{~K}(\mathrm{i})$, partial melting to ice II + liquid at $196 \mathrm{~K}$ (ii) and complete melting at $\sim 210 \mathrm{~K}$ (iii). Derivation of the ice liquidus is described in the text. The red arrow corresponds to the vertical path in $P, T$ space shown in supplementary Fig. S1 $(d)$.

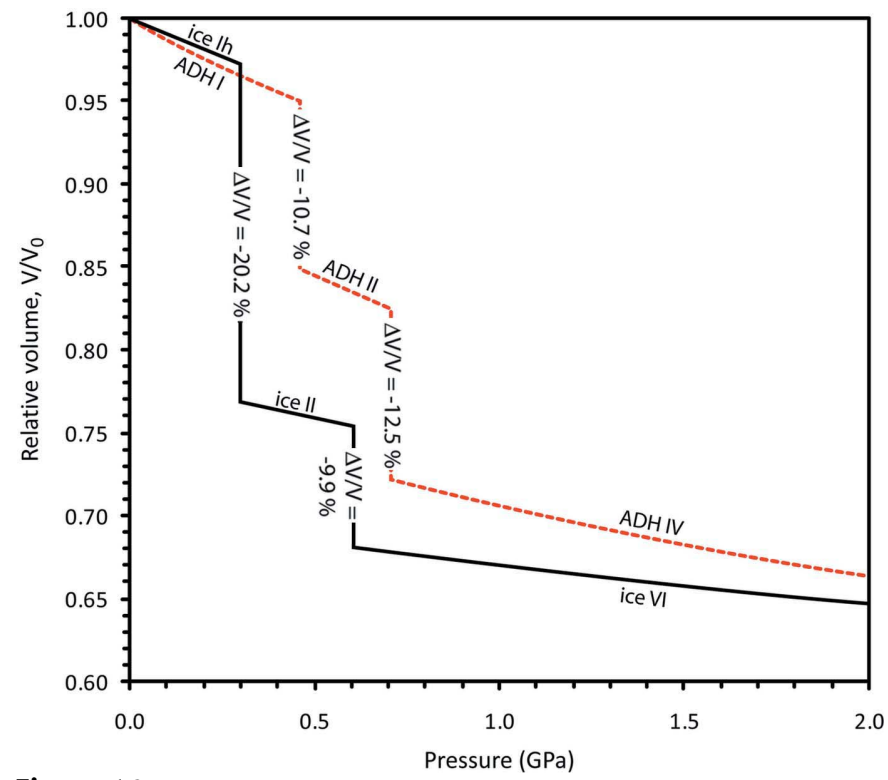

Figure 16

Relative compression $\left(V / V_{0}\right)$ curves for water ice (solid black line) and ammonia dihydrate (dashed red line) derived from the measurements reported in this paper, at temperatures of $185 \pm 15 \mathrm{~K}$. This summary plot shows that the volume contrasts through the two $\mathrm{ADH}$ phase transitions $(\mathrm{ADH} \mathrm{I} \rightarrow \mathrm{ADH}$ II and $\mathrm{ADH}$ II $\rightarrow$ ADH IV) are each smaller than those found in the water ice system (ice Ih $\rightarrow$ ice II and ice II $\rightarrow$ ice VI). However, the ADH polymorphs are all more compressible than the equivalent water ice phases at the same pressures; this is manifested as the steeper sub-horizontal lines. The combined effect is that both water ice and ADH experience similar overall degrees of densification $(\sim 35 \%)$ over the range $0<P<2 \mathrm{GPa}$. 


\section{Summary}

We have carried out neutron powder diffraction studies of ammonia hydrate + ice mixtures in the pressure range $0<P<$ $2 \mathrm{GPa}$ and the temperature range $150<T<240 \mathrm{~K}$. We have identified and characterized two new high-pressure polymorphs of ammonia dihydrate and one of ammonia monohydrate. We have also constrained the pressure-volume curves of ice polymorphs Ih, II, IX and VI at lower temperatures than most previous experimental studies and compared our results with available empirically and theoretically derived equations of state. We find excellent agreement with Feistel \& Wagner's (2006) equation of state for ice Ih, whereas the congruence with Choukroun \& Grasset (2007) is quite poor for ices I and IX. The TIP4P/2005-derived equation of state of Noya et al. (2007) gives very good agreement for ices Ih and VI, but poorer agreement for ice II, and extremely poor agreement for ice IX. For ice II, we have established the thermal expansion under pressure for the first time, allowing us to extend our previous equation of state (Fortes et al., 2005) with a term describing the temperature dependence of the bulk modulus.

We have presented data on the density of ADH polymorphs I, II and IV as a function of pressure up to $2 \mathrm{GPa}$, determining the density contrasts between these phases. We have also determined the thermal expansivity of ADH II in situ. The relative volume changes in water ice and ammonia dihydrate, in the range $0<P<2 \mathrm{GPa}$ and at temperatures of $185 \pm 15 \mathrm{~K}$, are summarized and compared in Fig. 16. The density of AMH II as a function of pressure has been constrained and compared with the known pressure-dependent density of AMH I to determine the density contrast between the two phases. The thermal expansivity of AMH II has been measured along two isobars, permitting a rough estimate of how the bulk modulus varies with temperature.

Lastly, observations of crystallization, and of melting, have allowed us to estimate crystal growth kinetic parameters and to infer the $P, T$ dependence of certain phase boundaries.

The authors gratefully acknowledge the STFC ISIS facility for beamtime to conduct these experiments, and would like to thank the following high-pressure technical staff, electrical engineers and cryostat support staff for their patience and hard work: Duncan Francis, Andy Church, Chris Goodway, John Dreyer and Jon Bones. The authors also acknowledge the assistance of Ms Helen Brand, Dr Peter Grindrod, Dr Katherine Joy and Dr Maria Alfredsson. This work was funded by fellowships awarded to ADF by the Science and Technology Facilities Council (STFC, ex-PPARC), grant Nos. PPA/P/S/2003/00247 and PP/E006515/1.

\section{References}

Arnold, G. P., Wenzel, R. P., Rabideau, S. W., Nereson, N. G. \& Bowman, A. L. (1971). J. Chem. Phys. 55, 589-595.

Avrami, M. (1939). J. Chem. Phys. 7, 1103-1112.

Avrami, M. (1940). J. Chem. Phys. 8, 212-224.

Bailey, I. F. (2003). Z. Kristallogr. 218, 84-95.
Bauer, M., Elsaesser, M. S., Winkel, K., Mayer, E. \& Loerting, T. (2008). Phys. Rev. B, 77, 220105.

Bergmann, J., Le Bail, A., Shirley, R. \& Zlokazov, V. (2004). Z. Kristallogr. 219, 783-790.

Bertie, J. E. \& Shehata, M. R. (1984). J. Chem. Phys. 81, 27-30.

Besson, J. M., Nelmes, R. J., Hamel, G., Loveday, J. S., Weill, G. \& Hull, S. (1992). Physica B, 180-181, 907-910.

Boone, S. C. (1989). PhD thesis, University of California, Los Angeles, USA.

Boone, S. \& Nicol, M. E. (1991). Proc. Lunar Planet. Sci. 21, 603-610. Boultif, A. \& Louër, D. (2004). J. Appl. Cryst. 37, 724-731.

Brand, H. E. A., Fortes, A. D., Wood, I. G., Knight, K. S. \& Vočadlo, L. (2009). Phys. Chem. Min. 36, 29-46.

Bridgman, P. W. (1935). J. Chem. Phys. 3, 597-605.

Chan, J. P. \& Giauque, W. F. (1964). J. Phys. Chem. 68, 3053-3057.

Choukroun, M. \& Grasset, O. (2007). J. Chem. Phys. 127, 124504.

Choukroun, M., Grasset, O., Le Menn, E., Morizet, Y. \& Tobie, G. (2007). Proc. Lunar Planet. Sci. 38, abstract 1606.

Croft, S. K., Lunine, J. I. \& Kargel, J. S. (1988). Icarus, 73, 279-293.

Cynn, H. C., Boone, S., Koumvakalis, A., Nicol, M. \& Stevenson, D. J. (1989). Proc. Lunar Planet. Sci. 19, 433-441.

Dantl, G. (1968). Phys. Condens. Matter, 7, 390-397.

Dyadin, Yu. A., Larionov, E. G., Aladko, E. Ya., Manakov, A. Yu., Zhurko, F. V., Mikina, T. V., Komarov, V. Yu. \& Grachev, E. V. (1999). J. Struct. Chem. 40, 790-795.

Feistel, R. \& Wagner, W. (2006). J. Chem. Ref. Data, 35, 1021-1047.

Finney, J. L. (1995). Acta Cryst. B51, 447-467.

Fortes, A. D. (2000). Icarus, 146, 444-452.

Fortes, A. (2004). PhD thesis, University of London, UK.

Fortes, A. D., Grindrod, P. M., Trickett, S. K. \& Vočadlo, L. (2007). Icarus, 188, 139-153.

Fortes, A. D., Wood, I. G., Alfredsson, M., Vočadlo, L. \& Knight, K. S. (2005). J. Appl. Cryst. 38, 612-618.

Fortes, A. D., Wood, I. G., Alfredsson, M., Vočadlo, L., Knight, K. S., Marshall, W. G., Tucker, M. G. \& Fernandez-Alonso, F. (2007). High Press. Res. 27, 201-212.

Fortes, A. D., Wood, I. G., Brodholt, J. P. \& Vočadlo, L. (2003). Icarus, 162, 59-73.

Fortes, A. D., Wood, I. G. \& Knight, K. S. (2008). Phys. Chem. Min. 35, 207-221.

Fortes, A. D., Wood, I. G., Knight, K. S., Brodholt, J. P., Alfredsson, M., McGrady, G. S. \& Vočadlo, L. (2003). J. Chem. Phys. 119, 10806-10813.

Gagnon, R. E., Kiefte, H., Clouter, M. J. \& Whalley, E. (1988). J. Chem. Phys. 89, 4522-4528.

Gagnon, R. E., Kiefte, H., Clouter, M. J. \& Whalley, E. (1990). J. Chem. Phys. 92, 1909-1914.

Grasset, O., Amiguet, E. \& Choukroun, M. (2005). High Press. Res. 25, 255-265.

Grasset, O. \& Pargamin, J. (2005). Planet. Space Sci. 53, 371-384.

Grasset, O. \& Sotin, C. (1996a). Proc. Lunar Planet. Sci. 27, 453-454.

Grasset, O. \& Sotin, C. (1996b). Icarus, 123, 101-112.

Grasset, O., Sotin, C. \& Dechamps, F. (2000). Planet. Space Sci. 48, 617-636.

Grindrod, P. M., Fortes, A. D., Nimmo, F., Feltham, D. L., Brodholt, J. P. \& Vočadlo, L. (2008). Icarus, 197, 137-151.

Gromnitskaya, E. L., Stal'gorova, O. V., Brazhkin, V. V. \& Lyapin, A. G. (2001). Phys. Rev. B, 64, 094205.

Hage, W., Hallbrucker, A., Mayer, E. \& Johari, G. P. (1994). J. Chem. Phys. 100, 2743-2747.

Hage, W., Hallbrucker, A., Mayer, E. \& Johari, G. P. (1995). J. Chem. Phys. 103, 545-550.

Hogenboom, D. L. \& Kargel, J. S. (1990). Proc. Lunar Planet. Sci. 21, 522.

Hogenboom, D. L., Kargel, J. S., Consolmagno, G. J., Holden, T. C., Lee, L. \& Buyyounouski, M. (1997). Icarus, 128, 171-180.

Hogenboom, D. L., Kargel, J. S., Holden, T. C. \& Buyyounouski, M. (1995). Proc. Lunar Planet. Sci. 26, 613-614. 
Hogenboom, D. L., Kargel, J. S., Holden, T. C. \& Ganasan, J. (1994). Proc. Lunar Planet. Sci. 25, 555-556.

Hogenboom, D. L., Winebrake, J., Consolmagno, G. J. \& Dalrymple, W. III (1989). Proc. Lunar Planet. Sci. 20, 420.

Ibberson, R. M., David, W. I. F. \& Knight, K. S. (1992). The High Resolution Neutron Powder Diffractometer (HRPD) at ISIS - A User Guide. Technical Report RAL-92-031. Rutherford Appleton Laboratory, Didcot, Oxfordshire, UK (http://www.isis.rl.ac.uk/ crystallography/documentation/HRPDguide/HRPDguideFramePage. htm).

ISIS Annual Report (1996). PEARL/HiPr, Dedicated Facility for High Pressure Diffraction. Technical Report RAL-TR-96-050, pp. 61-62. Rutherford Appleton Laboratory, Didcot, Oxfordshire, UK.

Johnson, M. L. \& Nicol, M. (1987). J. Geophys. Res. 92, 6339-6349.

Johnson, M. L., Schwake, A. \& Nicol, M. (1984). Proc. Lunar Planet. Sci. 15, 405-406.

Johnson, M. L., Schwake, A. \& Nicol, M. (1985). Ices in the Solar System, edited by J. Klinger, D. Benest, A. Dollfus \& R. Smoluchowski, pp. 39-47. Dordrecht: Reidel.

Johnson, W. A. \& Mehl, R. F. (1939). Trans. Am. Inst. Min. Metall. Pet. Eng. 135, 416-442.

Kahane, A., Klinger, J. \& Phillipe, M. (1969). Solid State Commun. 7, 1055-1056.

Kamb, B. (1965). Science, 150, 205-209.

Kaminsky, W. (2005). J. Appl. Cryst. 38, 566-567.

Kargel, J. S. (1992). Icarus, 100, 556-574.

Kargel, J. S., Croft, S. K., Lunine, J. I. \& Lewis, J. S. (1991). Icarus, 89, 93-112.

Kargel, J. S. \& Hogenboom, D. L. (1995). Proc. Lunar Planet. Sci. 26, 725-726.

Kolmogorov, A. N. (1937). Izv. Akad. Nauk SSSR Ser. Mater. 3, 355359.

Koumvakalis, A. (1988). PhD thesis, University of California, Los Angeles, USA.

Kuhs, W. F., Finney, J. L., Vettier, C. \& Bliss, D. V. (1984). J. Chem. Phys. 81, 3612-3623.

Kurnosov, A., Dubrovinsky, L., Kuznetsov, A. \& Dmitriev, V. (2006). Z. Naturforsch. Teil B, 61, 1573-1576.

Larsen, A. C. \& Von Dreele, R. B. (2000). General Structure Analysis System (GSAS). Report LAUR 86-748. Los Alamos National Laboratory, New Mexico, USA (http://www.ncnr.nist.gov/Xtal/ software/gsas.html).

Le Bail, A. (2005). Powder Diff. 20, 316-326.

Le Bail, A., Duroy, H. \& Fourquet, J. L. (1988). Mater. Res. Bull. 23, 447-452.

Leliwa-Kopystiński, J., Maruyama, M. \& Nakajima, T. (2002). Icarus, 159, 518-528.

Lewis, J. S. (1971). Icarus, 15, 174-185.

Lewis, J. S. (1972). Icarus, 16, 241-252.

Lewis, J. S. \& Prinn, R. G. (1980). Astrophys. J. 238, 357-364.

Lobban, C., Finney, J. L. \& Kuhs, W. F. (2000). J. Chem. Phys. 112, 7169-7180.

Lobban, C., Finney, J. L. \& Kuhs, W. F. (2002). J. Chem. Phys. 117, 3928-3934.

Londono, J. D., Finney, J. L. \& Kuhs, W. F. (1992). J. Chem. Phys. 97, 547-552.

Londono, J. D., Kuhs, W. F. \& Finney, J. L. (1993). J. Chem. Phys. 98, 4878-4888.
Lopes, R. M. C. et al. (2007). Icarus, 186, 395-412.

Lorenz, R. D., Stiles, B. W., Kirk, R. L., Allison, M. D., del Marmo, P. P., Iess, L., Lunine, J. I., Ostro, S. J. \& Hensley, S. (2008). Science, 319, 1649-1651.

Loveday, J. S. \& Nelmes, R. J. (1999). Phys. Rev. Lett. 83, 4329-4332. Loveday, J. S. \& Nelmes, R. J. (2004). High Press. Res. 24, 45-55.

Marshall, W. G. \& Francis, D. J. (2002). J. Appl. Cryst. 35, 122-125.

Mighell, A. D., Ondik, H. M. \& Molino, B. B. (1977). J. Phys. Chem. Ref. Data, 6, 675-829.

Mighell, A. D. \& Santoro, A. (1975). J. Appl. Cryst. 8, 372-374.

Mitzdorf, U. \& Helmreich, D. (1971). J. Acoust. Soc. Am. 49, 723 728.

Mousis, O., Gautier, D. \& Bocklée-Morvan, D. (2002). Icarus, 156, 162-175.

Mousis, O., Pargamin, J., Grasset, O. \& Sotin, C. (2002). Geophys. Res. Lett. 29, 2192.

Murnaghan, F. D. (1944). Proc. Natl Acad. Sci. USA, 30, 244-247.

Namand, S. C. \& Hockberger, P. E. (1992). Pflügers Arch. Eur. J. Phys. 420, 106-108.

Nelmes, R. J. \& Loveday, J. S. (1998). Diffraction Studies of the LowPressure Phases of Ammonia Hydrates. ISIS Experimental Report RB9411. CCLRC Rutherford Appleton Laboratory, Didcot, Oxfordshire, UK (http://www.isis.rl.ac.uk/ISIS98/reports/9411.pdf).

Nelmes, R. J., Loveday, J. S. \& Guthrie, M. (1999). Structural Changes Under Pressure in Ammonia Dihydrate and Ammonia Hemihydrate. ISIS Experimental Report RB 9859. CCLRC Rutherford Appleton Laboratory, Didcot, Oxfordshire, UK (http://www.isis. rl.ac.uk/ISIS99/reports/9859.pdf).

Noya, E. G., Menduiña, C., Aragones, J. L. \& Vega, C. (2007). J. Phys. Chem. 111, 15877-15888.

Pargamin, J., Mousis, O. \& Grasset, O. (2002). Proc. Lunar Planet. Sci. 33, abstract 1461.

Prinn, R. G. \& Fegley, B. Jr (1981). Astrophys. J. 249, 308-317.

Proctor, T. M. (1966). J. Acoust. Soc. Am. 39, 972-977.

Raulin, F. (2008). Space Sci. Rev. 135, 37-48.

Röttger, K., Endriss, A., Ihringer, J., Doyle, S. \& Kuhs, W. F. (1994). Acta Cryst. B50, 644-648.

Settle, M. (1979). Proc. Lunar Planet. Sci. 10, 1107-1109.

Shmyt'ko, I. M., Afonikova, N. S. \& Torgashev, V. I. (2002). Phys. Solid State, 44, 2309-2317.

Simakov, M. B. (2001). Proceedings of the First European Workshop on Exo/Astrobiology, ESA SP-496, pp. 211-214.

Smith, G. S. \& Snyder, R. L. (1979). J. Appl. Cryst. 12, 60-65.

Sohl, F., Hussmann, H., Schwentker, B., Spohn, T. \& Lorenz, R. D. (2003). J. Geophys. Res. Planets, 108, 5130-5143.

Telling, M. T. F. \& Andersen, K. H. (2005). Phys. Chem. Chem. Phys. 7, 1255-1261.

Telling, M. T. F. \& Andersen, K. H. (2008). The OSIRIS User Guide, 3rd ed., http://www.isis.rl.ac.uk/molecularSpectroscopy/osiris/.

Tobie, G., Grasset, O., Lunine, J. I., Mocquet, A. \& Sotin, C. (2005). Icarus, 175, 496-502.

Toby, B. H. (2001). J. Appl. Cryst. 34, 210-213.

Tulk, C. A., Kiefte, H., Clouter, M. J. \& Gagnon, R. E. (1997). J. Phys. Chem. B, 101, 6154-6157.

Wolff, P. M. de (1968). J. Appl. Cryst. 1, 108-113.

Yarger, J., Lunine, J. I. \& Burke, M. (1993). J. Geophys. Res. 98, 13109-13117.

Zheligovskaya, E. A. \& Malenkov, G. G. (2006). Russ. Chem. Rev. 75, 57-76. 